\title{
SECOND ORDER SUFFICIENT OPTIMALITY CONDITIONS FOR SOME STATE-CONSTRAINED CONTROL PROBLEMS OF SEMILINEAR ELLIPTIC EQUATIONS*
}

\author{
EDUARDO $\mathrm{CASAS}^{\dagger}$, FREDI TRÖLTZSCH ${ }^{\ddagger}$, AND ANDREAS UNGER ${ }^{\ddagger}$
}

\begin{abstract}
This paper deals with a class of optimal control problems governed by elliptic equations with nonlinear boundary condition. The case of boundary control is studied. Pointwise constraints on the control and certain equality and set-constraints on the state are considered. Second order sufficient conditions for local optimality of controls are established.
\end{abstract}

Key words. boundary control, semilinear elliptic equations, sufficient optimality conditions, state constraints

AMS subject classifications. 49K20, 35J25

PII. S0363012997324910

1. Introduction. In contrast to the optimal control of linear systems with a convex objective, where first order necessary optimality conditions are already sufficient for optimality, higher order conditions such as second order sufficient optimality conditions (SSC) should be employed to verify optimality for nonlinear systems. Second order sufficient optimality conditions have also proved to be useful for showing important properties of optimal control problems such as local uniqueness of optimal controls and their stability with respect to certain perturbations. Moreover, they may serve as an assumption to guarantee the convergence of numerical methods in optimal control. In this respect, we refer to the general expositions by Maurer and Zowe [15] and Maurer [14] for different aspects of second order sufficient optimality conditions. The approximation of programming problems in Banach spaces is discussed in Alt [2]. Moreover, Alt [3], [4] has established a general convergence analysis for Lagrange-Newton methods in Banach spaces.

Meanwhile, an extensive number of publications have been devoted to different aspects of second order sufficient optimality conditions for control problems governed by ordinary differential equations. The well-known two-norm discrepancy has in particular received a good deal of attention. We refer, for instance, to Ioffe [13] and Maurer [14].

First investigations of second order sufficient optimality conditions for control problems governed by partial differential equations have been published by Goldberg and Tröltzsch [11], [12] for the boundary control of parabolic equations with nonlinear boundary conditions. In [9], Casas, Tröltzsch, and Unger have extended these ideas to elliptic boundary control problems with pointwise constraints on the control. Moreover, they tightened the gap between second order necessary and sufficient

* Received by the editors July 21, 1997; accepted for publication (in revised form) December 4, 1998; published electronically May 11, 2000. This research was partially supported by European Union, under HCM project ERBCHRXCT940471, and by Deutsche Forschungsgemeinschaft, under project Tr 302/1-2. The research of the first author was also supported by Dirección General de Enseñanza Superior e Investigación Científica (Spain).

http://www.siam.org/journals/sicon/38-5/32491.html

${ }_{\dagger}^{\dagger}$ Departamento de Matemática Aplicada y Ciencias de la Computación, E.T.S.I. Industriales y de Telecomunicación, Universidad de Cantabria, 39071 Santander, Spain (casas@macc.unican.es).

${ }_{\ddagger}^{\ddagger}$ Fakultät für Mathematik, Technische Universität Chemnitz-Zwickau, D-09107 Chemnitz, Germany (f.troeltzsch@mathematik.tu-chemnitz.de, aug@shc.tcc-chemnitz.de). 
optimality conditions. This was done by the consideration of sets of strongly active constraints according to Dontchev et al. [10]. This technique is also related to first order sufficient optimality conditions introduced by Maurer and Zowe [15]. It should be mentioned that as many as four norms have to be used in this case $\left(L^{\infty}\right.$-norm for differentiation, $L^{2}$-norm to formulate second order sufficient optimality conditions, $L^{1}$-norm for the first order sufficient optimality condition, and certain $L^{p}$-norms to obtain optimal regularity results).

Bonnans [5] has shown that a very weak form of second order sufficient conditions can be used to verify local optimality for a particular class of semilinear elliptic control problems with constraints on the control: If the second order derivative of the Lagrange function is a Legendre form, then it suffices to have its positivity in all critical directions.

In our paper, the results of [9] will be extended to additional constraints on the state. In this way, we are continuing the investigations by Casas and Tröltzsch [8] on second order necessary conditions. We also rely on general ideas of Maurer and Zowe [15], combining their approach with a detailed splitting technique.

At the beginning, we aimed to establish second order sufficient optimality conditions for boundary control problems governed by semilinear elliptic equations in domains of arbitrary dimension with general pointwise constraints on the control and the state. However, we soon recognized that pointwise state-constraints lead to essential and somewhat surprising difficulties. To establish second order sufficient optimality conditions for problems with pointwise state-constraints given on the whole domain, we had to restrict ourselves to two-dimensional domains with controls appearing linearly in the boundary condition. These obstacles might indicate some limits for the "traditional" type of second order sufficient optimality conditions for control problems governed by PDEs.

If pointwise state-constraints are imposed on compact subsets of the domain, while the other quantities are sufficiently smooth, then arbitrary dimensions can be treated without restrictions on the nonlinearities. In this case the adjoint state belongs to $L^{\infty}(\Gamma)$. Moreover, we are able to avoid the assumption of linearity of the boundary condition with respect to the control by introducing some extended form of second order optimality conditions.

2. The optimal control problem. We consider the problem: Minimize the functional

$$
F_{0}(y, u)=\int_{\Omega} f(x, y(x)) d x+\int_{\Gamma} g(x, y(x), u(x)) d S(x)
$$

subject to the equation of state

$$
\left\{\begin{aligned}
-\Delta y(x)+y(x) & =0 & & \text { in } \Omega, \\
\partial_{\nu} y(x) & =b(x, y(x), u(x)) & & \text { on } \Gamma,
\end{aligned}\right.
$$

to the constraints on the state $y$

$$
\begin{aligned}
F_{i}(y) & =0, \quad i=1, \ldots, m, \\
E(y) & \in K,
\end{aligned}
$$

and to the constraints on the control $u$

$$
u_{a}(x) \leq u(x) \leq u_{b}(x) \quad \text { almost everywhere (a.e.) on } \Gamma .
$$


In this setting, $\Omega \subset \mathbb{R}^{n}$ is a bounded domain with a Lipschitz boundary $\Gamma$ according to the definition by Nečas [17]. Moreover, sufficiently smooth functions $f: \Omega \times \mathbb{R} \rightarrow \mathbb{R}$ and $g, b: \Gamma \times \mathbb{R}^{2} \rightarrow \mathbb{R}$ are given. The symbol $\partial_{\nu}$ is used for the derivative in the direction of the unit outward normal $\nu$ on $\Gamma$. The functionals $F_{i}: C(\bar{\Omega}) \rightarrow \mathbb{R}$, $i=1, \ldots, m$, are supposed to be twice continuously Fréchet differentiable, that is, to be of class $C^{2}$. By $E$ we denote a mapping of class $C^{2}$ from $C(\bar{\Omega})$ into a real Banach space $Z$. $K \subset Z$ is a nonempty convex closed set, and $u_{a}, u_{b}: \Gamma \rightarrow \mathbb{R}$ are functions of $L^{\infty}(\Gamma)$ satisfying $u_{a}(x) \leq u_{b}(x)$ on $\Gamma$.

The control $u$ is looked for in the control space $\mathcal{U}=L^{\infty}(\Gamma)$, while the state $y$ is defined as a weak solution of (2.2) in the state space $C(\bar{\Omega}) \cap H^{1}(\Omega)=Y$, that is,

$$
\int_{\Omega}(\nabla y \nabla v+y v) d x=\int_{\Gamma} b(\cdot, y, u) v d S \quad \forall v \in H^{1}(\Omega) .
$$

We endow $Y$ with the norm $\|y\|_{Y}=\|y\|_{C(\bar{\Omega})}+\|y\|_{H^{1}(\Omega)}$. The following assumptions are imposed on the given quantities.

(A1) For each fixed $x \in \Omega$ or $\Gamma$, respectively, the functions $f=f(x, y), g=$ $g(x, y, u)$, and $b=b(x, y, u)$ are of class $C^{2}$ with respect to $(y, u)$. For fixed $(y, u)$, they are Lebesgue measurable with respect to $x \in \Omega$ or $x \in \Gamma$, respectively.

Throughout the paper, partial derivatives are indicated by associated subscripts. For instance, $b_{y u}$ stands for $\partial^{2} b / \partial y \partial u$. By $b^{\prime}(x, y, u)$ and $b^{\prime \prime}(x, y, u)$ we denote the gradient and the Hessian matrix of $b$ with respect to $(y, u)$ :

$$
b^{\prime}(x, y, u)=\left(\begin{array}{c}
b_{y}(x, y, u) \\
b_{u}(x, y, u)
\end{array}\right), \quad b^{\prime \prime}(x, y, u)=\left(\begin{array}{cc}
b_{y y}(x, y, u) & b_{y u}(x, y, u) \\
b_{u y}(x, y, u) & b_{u u}(x, y, u)
\end{array}\right)
$$

$\left|b^{\prime}\right|$ and $\left|b^{\prime \prime}\right|$ are defined by adding the absolute values of all entries.

In the next assumption, fixed parameters $p>n-1$ and $s, r$ are used, which depend on $n$. For the possible (minimal) choice of $s$ and $r$ we refer to the discussion of regularity in (3.13). Roughly speaking, we have $\left.y\right|_{\Gamma} \in L^{s}(\Gamma)$ and $y \in L^{r}(\Omega)$ in the linearized system (2.2) if $u \in L^{2}(\Gamma)$. As usual, $s^{\prime}$ and $r^{\prime}$ denote conjugate numbers. For instance, $s^{\prime}$ is defined by $1 / s^{\prime}+1 / s=1$.

(A2) For all $M>0$ there are constants $C_{M}>0$, functions $\Psi_{f}^{M} \in L^{(r / 2)^{\prime}}(\Omega)$, $\Psi_{g}^{M, 1} \in L^{(s / 2)^{\prime}}(\Gamma), \Psi_{g}^{M, 2} \in L^{2(s / 2)^{\prime}}(\Gamma), \Psi_{g}^{M, 3} \in L^{\infty}(\Gamma)$, and a continuous, monotone increasing function $\eta \in C\left(\mathbb{R}^{+} \cup\{0\}\right)$ with $\eta(0)=0$ such that

(i)

$$
b_{y}(x, y, u) \leq 0 \quad \text { a.e. } x \in \Gamma, \forall(y, u) \in \mathbb{R}^{2},
$$

$b(\cdot, 0,0) \in L^{p}(\Gamma)$, for a $p>n-1$,

$\left|b^{\prime}(x, y, u)\right|+\left|b^{\prime \prime}(x, y, u)\right| \leq C_{M}$,

$\left|b^{\prime \prime}\left(x, y_{1}, u_{1}\right)-b^{\prime \prime}\left(x, y_{2}, u_{2}\right)\right| \leq C_{M} \eta\left(\left|y_{1}-y_{2}\right|+\left|u_{1}-u_{2}\right|\right)$

for almost all $x \in \Gamma$ and all $|y|,|u|,\left|y_{i}\right|,\left|u_{i}\right| \leq M, i=1,2$;

(ii) $f(\cdot, 0) \in L^{1}(\Omega), f_{y}(\cdot, 0) \in L^{r^{\prime}}(\Omega), f_{y y}(\cdot, 0) \in L^{(r / 2)^{\prime}}(\Omega)$

$\left|f_{y y}\left(x, y_{1}\right)-f_{y y}\left(x, y_{2}\right)\right| \leq \Psi_{f}^{M}(x) \eta\left(\left|y_{1}-y_{2}\right|\right)$

$\forall x \in \Omega,\left|y_{i}\right| \leq M, i=1,2$; 
(iii) $g(\cdot, 0,0) \in L^{1}(\Gamma), g_{y}(\cdot, 0,0) \in L^{s^{\prime}}(\Gamma), g_{u}(\cdot, 0,0) \in L^{2}(\Gamma)$, $g_{y y}(\cdot, 0,0) \in L^{(s / 2)^{\prime}}(\Gamma), g_{y u}(\cdot, 0,0) \in L^{2(s / 2)^{\prime}}(\Gamma), g_{u u}(\cdot, 0,0) \in L^{\infty}(\Gamma)$ (here, $\cdot$ stands for $x$ ) $\left|g_{y y}\left(x, y_{1}, u_{1}\right)-g_{y y}\left(x, y_{2}, u_{2}\right)\right| \leq \Psi_{g}^{M, 1}(x) \eta\left(\left|y_{1}-y_{2}\right|+\left|u_{1}-u_{2}\right|\right)$, $\left|g_{y u}\left(x, y_{1}, u_{1}\right)-g_{y u}\left(x, y_{2}, u_{2}\right)\right| \leq \Psi_{g}^{M, 2}(x) \eta\left(\left|y_{1}-y_{2}\right|+\left|u_{1}-u_{2}\right|\right)$, $\left|g_{u u}\left(x, y_{1}, u_{1}\right)-g_{u u}\left(x, y_{2}, u_{2}\right)\right| \leq \Psi_{g}^{M, 3}(x) \eta\left(\left|y_{1}-y_{2}\right|+\left|u_{1}-u_{2}\right|\right)$, for almost all $x \in \Gamma$ and all $\left|y_{i}\right| \leq M,\left|u_{i}\right| \leq M$.

Remark 2.1. Notice that the estimates in (i)-(iii) imply boundedness and Lipschitz properties of $b, f, g, b^{\prime}, f^{\prime}, g^{\prime}$ in several $L$-spaces. We omit them, because they follow from the mean value theorem.

(A3) (i) Let us define the norm

$$
\|y\|_{2}=\|y\|_{C(A)}+\|y\|_{L^{r}(\Omega)}+\|y\|_{L^{s}(\Gamma)}
$$

for $y \in C(\bar{\Omega})$, where $A \subset \bar{\Omega}$ is a certain measurable compact subset. Here $A$ stands for a set, where we know $y \in C(A)$ for Neumann boundary data given in $L^{2}(\Gamma)$. In the case $n=2$ we may take $A=\bar{\Omega}$, while $A \subset \Omega$ is needed for $n>2$. For $A=\emptyset$ we put $\|y\|_{C(A)}=0$.

We assume at a fixed reference state $\bar{y} \in C(\bar{\Omega})$ that

$$
\begin{aligned}
\left|F_{i}^{\prime}(\bar{y}) y\right| & \leq C_{F}\|y\|_{2} \quad \forall y \in C(\bar{\Omega}), \\
\left|F_{i}^{\prime \prime}(\bar{y})\left[y_{1}, y_{2}\right]\right| & \leq C_{F}\left\|y_{1}\right\|_{2}\left\|y_{2}\right\|_{2} \quad \forall y_{1}, y_{2} \in C(\bar{\Omega})
\end{aligned}
$$

holds with some $C_{F}>0$. Moreover, we require with a $C_{M}>0$

$$
\begin{aligned}
\left|F_{i}^{\prime}\left(y_{1}\right) y-F_{i}^{\prime}\left(y_{2}\right) y\right| & \leq C_{M}\left\|y_{1}-y_{2}\right\|_{2}\|y\|_{2}, \\
\left|\left(F_{i}^{\prime \prime}\left(y_{1}\right)-F_{i}^{\prime \prime}\left(y_{2}\right)\right)[y, v]\right| & \leq C_{M} \eta\left(\left\|y_{1}-y_{2}\right\|_{C(\bar{\Omega})}\right)\|y\|_{2}\|v\|_{2}
\end{aligned}
$$

$\forall y_{j}$ with $\left\|y_{j}\right\|_{C(\bar{\Omega})} \leq M, j=1,2, \forall y, v$ from $C(\bar{\Omega})$, and $\forall i=1, \ldots, m$.

(ii) Analogous assumptions are imposed on $E: C(\bar{\Omega}) \rightarrow Z$, where $\|\cdot\|_{Z}$ is to be substituted for $|\cdot|$. For instance,

$$
\left\|E^{\prime}(\bar{y}) y\right\|_{Z} \leq C_{E}\|y\|_{2} \quad \forall y \in C(\bar{\Omega})
$$

is supposed.

We shall explain the main constructions of our paper by the following canonical example $(\mathrm{P})$ that fits in the general setting.

Example (P). Minimize

$$
\frac{1}{2} \int_{\Omega}\left(y-y_{d}\right)^{2} d x+\frac{\alpha}{2} \int_{\Gamma} u^{2} d S
$$

subject to

$$
\begin{aligned}
-\triangle y+y & =0 & & \text { in } \Omega, \\
\partial_{\nu} y & =u-y^{3} & & \text { on } \Gamma,
\end{aligned}
$$

and

$$
|u| \leq 1, \quad y(0) \leq y_{0}
$$

in the open unit ball $\Omega \subset \mathbb{R}^{3}$ around zero, where $\alpha>0, y_{0} \in \mathbb{R}$, and $y_{d} \in$ $L^{\infty}(\Omega)$ are given. Here, we have $Z=\mathbb{R}, K=\mathbb{R}^{-}, A=\{0\}, E(y)=y(0)-y_{0}$, and we need $y \in C(\Omega)$ to make $E$ well defined. 
3. The state equation and first order necessary optimality conditions. It can be shown that the equation $(2.2)$ admits for each $u \in \mathcal{U}^{a d}$ a unique weak solution $y=y(u) \in Y$, where $\mathcal{U}^{a d}=\left\{u \in L^{\infty}(\Gamma) \mid u_{a}(x) \leq u(x) \leq u_{b}(x)\right.$ a.e. on $\left.\Gamma\right\}$. Moreover, there is a constant $M$ such that

$$
\|y(u)\|_{Y} \leq M \quad \forall u \in \mathcal{U}^{a d} .
$$

In particular, it holds that $\|y\|_{C(\bar{\Omega})} \leq M$. Casas and Tröltzsch [8] have proved that the mapping $u \mapsto y(u)$ from $L^{\infty}(\Gamma)$ into $Y$ is of class $C^{2}$. Furthermore, the Lipschitz property

$$
\left\|y\left(u_{1}\right)-y\left(u_{2}\right)\right\|_{2} \leq C_{2}\left\|u_{1}-u_{2}\right\|_{L^{2}(\Gamma)}
$$

holds for all $u_{1}, u_{2} \in \mathcal{U}^{a d}$, where $C_{2}$ is a positive constant and $\|\cdot\|_{2}$ is defined in (A3). For fixed $u \in \mathcal{U}^{a d}$ we have $b(\cdot, y, u) \in L^{p}(\Gamma)$, hence the weak solution $y \in Y$ of $(2.2)$ belongs to the space

$$
Y_{q, p}=\left\{y \in H^{1}(\Omega) \mid-\Delta y+y \in L^{q}(\Omega), \partial_{\nu} y \in L^{p}(\Gamma)\right\},
$$

which is known to be continuously embedded into $Y=C(\bar{\Omega}) \cap H^{1}(\Omega)$ for each $q>n / 2$ and each $p>n-1$.

In all of what follows we assume that a reference pair $(\bar{y}, \bar{u}) \in Y \times \mathcal{U}^{\text {ad }}$ is given, satisfying, together with an associated adjoint state $\bar{\varphi} \in W^{1, \sigma}(\Omega) \forall \sigma<n /(n-1)$, and with Lagrange multipliers

$$
\bar{\lambda}=\left(\bar{\lambda}_{1}, \ldots, \bar{\lambda}_{m}\right)^{T} \in \mathbb{R}^{m}, \quad \bar{z}^{*} \in Z^{*},
$$

the associated standard first order necessary optimality conditions. We will just assume them. They can be proved following Casas [7], Bonnans and Casas [6], or Zowe and Kurcyusz [23]. The first order optimality system to be satisfied by $(\bar{y}, \bar{u})$ consists of the state equations (2.2), the constraint $\bar{u} \in \mathcal{U}^{a d}$, the adjoint equations

$$
\begin{aligned}
-\Delta \bar{\varphi}+\bar{\varphi} & =f_{y}(\cdot, \bar{y})+\left.\sum_{i=1}^{m} \bar{\lambda}_{i} F_{i}^{\prime}(\bar{y})\right|_{\Omega}+\left.\left(E^{\prime} \bar{y}\right)^{*} \bar{z}^{*}\right|_{\Omega} \quad \text { in } \Omega \\
\partial_{\nu} \bar{\varphi} & =b_{y}(\cdot, \bar{y}, \bar{u}) \bar{\varphi}+g_{y}(\cdot, \bar{y}, \bar{u})+\left.\sum_{i=1}^{m} \bar{\lambda}_{i} F_{i}^{\prime}(\bar{y})\right|_{\Gamma}+\left.\left(E^{\prime} \bar{y}\right)^{*} \bar{z}^{*}\right|_{\Gamma} \quad \text { on } \Gamma
\end{aligned}
$$

for the adjoint state $\bar{\varphi}$, the complementary slackness condition

$$
\left\langle\bar{z}^{*}, \kappa-E(\bar{y})\right\rangle \leq 0 \quad \forall \kappa \in K,
$$

and the variational inequality

$$
\int_{\Gamma}\left(g_{u}(x, \bar{y}(x), \bar{u}(x))+\bar{\varphi}(x) b_{u}(x, \bar{y}(x), \bar{u}(x))\right)(u(x)-\bar{u}(x)) d S(x) \geq 0
$$

$\forall u \in \mathcal{U}^{a d}$. We have $F_{i}^{\prime}(\bar{y}) \in C(\bar{\Omega})^{*}, i=1, \ldots, m$, and $E^{\prime}(\bar{y})^{*} \bar{z}^{*} \in C(\bar{\Omega})^{*}$; hence these quantities can be identified with real Borel measures on $\bar{\Omega}$. Let a nonnegative function $\beta \in L^{\infty}(\Gamma)$ and real Borel measures $\mu_{\Omega}$ and $\mu_{\Gamma}$ concentrated on $\Omega$ and $\Gamma$, respectively, be given. Then the problem

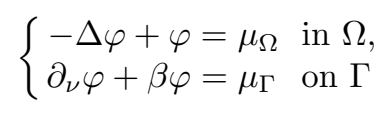


admits a unique solution $\varphi \in W^{1, \sigma}(\Omega) \forall \sigma<n /(n-1)$ (see Casas [7]; the reader is also referred to Stampacchia [20] for the Dirichlet case). In view of this, we may write

$$
\bar{\varphi}=\varphi_{0}+\sum_{i=1}^{m} \lambda_{i} \varphi_{i}+\varphi_{E}
$$

where $\varphi_{0}, \varphi_{i}$, and $\varphi_{E}$ solve (3.6) for $\mu_{\Omega}=f_{y},\left.F_{i}^{\prime}(\bar{y})\right|_{\Omega},\left.E^{\prime}(\bar{y})^{*} \bar{z}^{*}\right|_{\Omega}$, and $\mu_{\Gamma}=g_{y}$, $\left.F_{i}^{\prime}(\bar{y})\right|_{\Gamma},\left.E^{\prime}(\bar{y})^{*} \bar{z}^{*}\right|_{\Gamma}$, respectively. We have at least $\varphi_{0}, \varphi_{i}$, and $\varphi_{E}$ in $W^{1, \sigma}(\Omega)$. Moreover, $\bar{\varphi}$ satisfies the formula of integration by parts

$$
\int_{\Omega}(-\Delta y+y) \varphi d x+\int_{\Gamma}\left(\partial_{\nu} y+\beta y\right) \varphi d S(x)=\int_{\Omega} y d \mu_{\Omega}+\int_{\Gamma} y d \mu_{\Gamma}
$$

$\forall y \in Y_{q, p}$, where $q>n / 2, p>n-1$. It is easy to verify that the optimality conditions can be expressed by the Lagrange function

$$
\begin{aligned}
\mathcal{L}\left(y, u, \varphi, \lambda, z^{*}\right)=F_{0}(y, u) & -\int_{\Omega}(-\Delta y+y) \varphi d x-\int_{\Gamma}\left(\partial_{\nu} y-b(\cdot, y, u)\right) \varphi d S \\
& +\sum_{j=1}^{m} \lambda_{j} F_{j}(y)+\left\langle z^{*}, E(y)\right\rangle
\end{aligned}
$$

$\mathcal{L}: Y_{q, p} \times \mathcal{U} \times W^{1, \sigma}(\Omega) \times \mathbb{R}^{m} \times Z^{*} \rightarrow \mathbb{R}$. The regularity of $y$ and $\varphi$ fits together, as $\varphi \in W^{1, \sigma}(\Omega) \forall \sigma<n /(n-1)$ ensures $\varphi \in L^{s}(\Omega) \forall s<n /(n-2)$ (Nečas [17, Thm. 3.4, p. 69]), and $\left.\varphi\right|_{\Gamma} \in L^{r}(\Gamma)$ holds $\forall r<1+1 /(n-2)$ [17, Thm. 4.2, p. $84])$. Therefore, this definition makes sense. In $(3.8),\langle\cdot, \cdot\rangle$ denotes the duality pairing between $Z$ and its dual space $Z^{*}$. The Lagrange function $\mathcal{L}$ is of class $C^{2}$ with respect to $(y, u)$ for fixed $\varphi, \lambda$, and $z^{*}$.

Thanks to (3.7), the optimality system can be rewritten in terms of $\mathcal{L}$. Then it is expressed by (2.6), the constraints on the state (2.3), (2.4), the constraints on the control $u \in \mathcal{U}^{a d}$, and

$$
\begin{aligned}
\mathcal{L}_{y}\left(\bar{y}, \bar{u}, \bar{\varphi}, \bar{\lambda}, \bar{z}^{*}\right) y & =0 & & \forall y \in Y, \\
\mathcal{L}_{u}\left(\bar{y}, \bar{u}, \bar{\varphi}, \bar{\lambda}, \bar{z}^{*}\right)(u-\bar{u}) & \geq 0 & & \forall u \in \mathcal{U}^{a d}, \\
\left\langle\bar{z}^{*}, \kappa-E(\bar{y})\right\rangle & \leq 0 & & \forall \kappa \in K .
\end{aligned}
$$

This form is more convenient for our later evaluations.

Example. In (P), adjoint equation and variational inequality are given by

$$
\begin{array}{cl}
-\triangle \bar{\varphi}+\bar{\varphi}=\bar{y}-y_{d}+\bar{z}^{*} \circ \delta(0), & \partial_{\nu} \bar{\varphi}+3 \bar{y}^{2} \bar{\varphi}=0, \\
\int_{\Gamma}(\alpha \bar{u}+\bar{\varphi})(u-\bar{u}) d S \geq 0 & \forall|u| \leq 1,
\end{array}
$$

where $\delta(0)$ is the Dirac measure.

To shorten our notation, derivatives taken at $\left(\bar{y}, \bar{u}, \bar{\varphi}, \bar{\lambda}, \bar{z}^{*}\right)$ will be indicated by a bar. For instance, $\overline{\mathcal{L}}_{y} y, \overline{\mathcal{L}}_{u}(u-\bar{u})$ stand for the derivatives in (3.9) and (3.10), respectively. $\overline{\mathcal{L}}_{y y}\left[y_{1}, y_{2}\right]$ denotes the second order derivative of $\mathcal{L}$ in the directions $y_{1}, y_{2}$ taken at $\left(\bar{y}, \bar{u}, \bar{\varphi}, \bar{\lambda}, \bar{z}^{*}\right)$. Moreover, $\overline{\mathcal{L}}_{w w}\left[w_{1}, w_{2}\right]$ is the second order derivative of $\mathcal{L}$ in the directions $w_{1}=\left(y_{1}, u_{1}\right), w_{2}=\left(y_{2}, u_{2}\right)$. If $w_{1}=w_{2}=w$, then we write for short $\overline{\mathcal{L}}_{w w}[w, w]=\overline{\mathcal{L}}_{w w}[w]^{2}$. 
Next we provide some useful results on linearized versions of the state equation. Regard first the linear system

$$
\begin{cases}-\Delta y+y=f & \text { in } \Omega \\ \partial_{\nu} y+\beta y=g & \text { on } \Gamma\end{cases}
$$

where $\beta \in L^{\infty}(\Gamma)$ is nonnegative. For each pair $(f, g) \in L^{1}(\Omega) \times L^{1}(\Gamma)$, this system admits a unique solution $y \in W^{1, \sigma}(\Omega)$, where $\sigma<n /(n-1)$; see Casas [7]. (Notice that a function of $L^{1}$ can be considered as a Borel measure.) On the other hand, the solution $y$ of (3.12) belongs to $H^{1}(\Omega) \cap C(\bar{\Omega})$ if $(f, g) \in L^{q}(\Omega) \times L^{p}(\Gamma)$. This regularity result is well known for domains with $C^{1}$-boundary. Moreover, it remains true for domains with Lipschitz boundary in the sense of Nečas [17] (see Stampacchia [19] and Murthy and Stampacchia [16]). On account of this, the mapping $D:(f, g) \mapsto$ $\left(y,\left.y\right|_{\Gamma}\right)$ is continuous from $L^{1}(\Omega) \times L^{1}(\Gamma)$ into $L^{s}(\Omega) \times L^{t}(\Gamma)$ for $s<n /(n-2)$ and $t<(n-1) /(n-2)$. $D$ is continuous also from $L^{q}(\Omega) \times L^{p}(\Gamma)$ into $L^{\infty}(\Omega) \times L^{\infty}(\Gamma)$. We obtain these spaces by embedding results for $W^{1, \sigma}(\Omega)$ [1], [17], [20]. In both cases, this mapping is linear and continuous. Interpolation theory applies to show the following results for $D$ considered as a mapping defined on $L^{2}(\Omega) \times L^{2}(\Gamma)$ :

$$
y \in\left\{\begin{array} { l r } 
{ C ( \overline { \Omega } ) , } & { n = 2 , } \\
{ L ^ { r } ( \Omega ) \forall r < \infty , } & { n = 3 , } \\
{ L ^ { r } ( \Omega ) \forall r < \frac { 2 n } { n - 3 } , } & { n \geq 4 , }
\end{array} \quad y | _ { \Gamma } \in \left\{\begin{array}{ll}
C(\Gamma), & n=2, \\
L^{s}(\Gamma) \forall s<\infty, & n=3, \\
L^{s}(\Gamma) \forall s<\frac{2(n-1)}{n-3}, & n \geq 4 .
\end{array}\right.\right.
$$

4. Regularity condition and linearization theorem. Let us recall that we consider a fixed reference pair $(\bar{y}, \bar{u})$ satisfying, together with $\left(\bar{\varphi}, \bar{\lambda}, \bar{z}^{*}\right)$, the first order necessary conditions (3.9)-(3.11).

The linearized cone of $\mathcal{U}^{a d}$ at $\bar{u}$ is the set $\mathcal{C}(\bar{u})=\left\{v \in L^{\infty}(\Gamma) \mid v=\varrho(u-\bar{u}), \varrho \geq\right.$ $\left.0, u \in \mathcal{U}^{a d}\right\}$. Let $F=F(y)$ denote the mapping $y \mapsto\left(F_{1}(y), \ldots, F_{m}(y)\right)^{T}$ from $Y$ to $\mathbb{R}^{m}$. For convenience, we introduce the set of all feasible pairs

$$
\mathcal{M}=\left\{w=(y, u) \in Y \times \mathcal{U}^{a d} \mid y=G(u) \text { and } y \text { satisfies the state-constraints }\right\}
$$

(notice that $G$ is the nonlinear control-state-mapping). Following Maurer and Zowe [15], the linearized cone $L(\mathcal{M}, \bar{w})$ at $\bar{w}=(\bar{y}, \bar{u})$ is defined by

$$
L(\mathcal{M}, \bar{w})=\{w \mid w=(y, u), u \in \mathcal{C}(\bar{u}) \text { and }(y, u) \text { satisfies }(4.1)-(4.3)\},
$$

where

$$
\left\{\begin{array}{rlrl}
-\Delta y+y & =0 & & \text { in } \Omega, \\
\partial_{\nu} y & =b_{y}(\cdot, \bar{y}, \bar{u}) y+b_{u}(\cdot, \bar{y}, \bar{u}) u & & \text { on } \Gamma, \\
F^{\prime}(\bar{y}) y & =0 \\
E^{\prime}(\bar{y}) y & \in K(E(\bar{y})) . & &
\end{array}\right.
$$

Here, $K(E(\bar{y}))=\{z \in Z \mid z=\varrho(\kappa-E(\bar{y})), \varrho \geq 0, \kappa \in K\}$ is the conical hull of $K-E(\bar{y})$.

REMARK 4.1. The choice $Z=\mathbb{R}^{k}, E(y)=\left(E_{1}(y), \ldots, E_{k}(y)\right)^{T}, K=\left(\mathbb{R}^{k}\right)^{-}$for $E: Y \rightarrow Z$ is of particular interest. Then (4.3) reduces to

$$
E_{i}^{\prime}(\bar{y}) y \leq 0
$$

for all active $i \in\{1, \ldots, k\}$, that is for all $i$, where $E_{i}(\bar{y})=0$ holds. 
Example. The linearized cone for $(\mathrm{P})$ is the set of the following pairs $(y, u)$ : They satisfy $u \in \mathcal{C}(\bar{u})$ and

$$
\begin{gathered}
-\triangle y+y=0, \quad \partial_{\nu} y+3 \bar{y}^{2} y=u, \\
y(0) \leq 0,
\end{gathered}
$$

if $\bar{y}(0)=y_{0}$ (active state constraint). If the state constraint is not active, then (4.5) disappears.

The following regularity assumption $(\mathrm{R})$ is basic for our further analysis: To formulate $(\mathrm{R})$ we combine the two state constraints to one general constraint. We therefore take $\boldsymbol{Z}=\mathbb{R}^{m} \times Z, \boldsymbol{K}=\{0\} \times K$, define $T: Y \rightarrow \boldsymbol{Z}$ by $T(y)=(F(y), E(y))$, and put $\boldsymbol{K}(T(\bar{y}))=\{0\} \times K(E(\bar{y}))$. The regularity condition was introduced by Zowe and Kurcyusz [23] and requires

$$
T^{\prime}(\bar{y}) G^{\prime}(\bar{u}) \mathcal{C}(\bar{u})-\boldsymbol{K}(T(\bar{y}))=\boldsymbol{Z} .
$$

This condition is sufficient for the existence of a (nondegenerate) Lagrange multiplier associated to the state-constraint $E(y) \in K$; see [23]. We should emphasize that $(\mathrm{R})$ does not rely on the condition int $K \neq \emptyset$. In Appendix 7.1 we shall present some sufficient conditions for (R) which, however, require int $K \neq \emptyset$. (R) is discussed for the canonical example (P) there. For $Z=\mathbb{R}^{k}, K=\left(\mathbb{R}^{k}\right)^{-}$, the condition (R) is equivalent to the well-known Mangasarian-Fromowitz condition.

TheOREm 4.2. Suppose that $(R)$ is satisfied. Then for all pairs $(\hat{y}, \hat{u}) \in \mathcal{M}$ there is a pair $(y, u) \in L(\mathcal{M}, \bar{w})$ such that the difference $r=\left(r^{y}, r^{u}\right)=(\hat{y}, \hat{u})-(\bar{y}, \bar{u})-(y, u)$ can be estimated by

$$
\begin{aligned}
\|r\|_{Y \times L^{\infty}(\Gamma)} & \leq C_{L, p}\|\hat{u}-\bar{u}\|_{L^{\infty}(\Gamma)}\|\hat{u}-\bar{u}\|_{L^{p}(\Gamma)} \quad \forall p>n-1, \\
\|r\| & \leq C_{L, 2}\|\hat{u}-\bar{u}\|_{L^{\infty}(\Gamma)}\|\hat{u}-\bar{u}\|_{L^{2}(\Gamma)},
\end{aligned}
$$

where $\|r\|=\left\|r^{y}\right\|_{2}+\left\|r^{u}\right\|_{L^{2}(\Gamma)}$. In the particular case $b(x, y, u)=b_{1}(x, y)+b_{2}(x) u$ we have

$$
\|r\|_{Y \times L^{\infty}(\Gamma)} \leq C_{L, p}\|\hat{u}-\bar{u}\|_{L^{p}(\Gamma)}^{2} \quad \forall p>n-1 .
$$

This theorem is proved in Appendix 7.2. Let us conclude this section by considering some useful estimates for $\mathcal{L}^{\prime \prime}$ and for certain remainder terms. First, we evaluate

$$
\overline{\mathcal{L}}^{\prime \prime}\left[\left(y_{1}, u_{1}\right),\left(y_{2}, u_{2}\right)\right]=\mathcal{L}^{\prime \prime}\left(\bar{y}, \bar{u}, \bar{\varphi}, \bar{\lambda}, \bar{z}^{*}\right)\left[\left(y_{1}, u_{1}\right),\left(y_{2}, u_{2}\right)\right],
$$

where $\mathcal{L}^{\prime \prime}$ denotes the second order derivative of $\mathcal{L}$ with respect to $(y, u)$. We have

$$
\begin{aligned}
\overline{\mathcal{L}}^{\prime \prime}\left[\left(y_{1}, u_{1}\right),\left(y_{2}, u_{2}\right)\right]= & \int_{\Omega} f_{y y}(\cdot, \bar{y}) y_{1} y_{2} d x+\int_{\Gamma}\left(y_{1}, u_{1}\right) g^{\prime \prime}(\cdot, \bar{y}, \bar{u})\left(y_{2}, u_{2}\right)^{T} d S \\
& +\int_{\Gamma} \bar{\varphi} \cdot\left(y_{1}, u_{1}\right) b^{\prime \prime}(\cdot, \bar{y}, \bar{u})\left(y_{2}, u_{2}\right)^{T} d S \\
& +\sum_{i=1}^{m} \bar{\lambda}_{i} F_{i}^{\prime \prime}(\bar{y})\left[y_{1}, y_{2}\right]+\left\langle\bar{z}^{*}, E^{\prime \prime}(\bar{y})\left[y_{1}, y_{2}\right]\right\rangle .
\end{aligned}
$$


Example. In the case of $(\mathbf{P}), \overline{\mathcal{L}}^{\prime \prime}$ admits the form

$$
\overline{\mathcal{L}}^{\prime \prime}\left[\left(y_{1}, u_{1}\right),\left(y_{2}, u_{2}\right)\right]=\int_{\Omega} y_{1} y_{2} d x+\int_{\Gamma}\left(6 \bar{\varphi} \bar{y} y_{1} y_{2}+\alpha u_{1} u_{2}\right) d S .
$$

The term connected with $\bar{\varphi}$ causes trouble, more precisely,

$$
I=\int_{\Gamma} \bar{\varphi}\left(b_{y y}(\cdot, \bar{y}, \bar{u}) y_{1} y_{2}+b_{y u}(\cdot, \bar{y}, \bar{u})\left(y_{1} u_{2}+y_{2} u_{1}\right)+b_{u u}(\cdot, \bar{y}, \bar{u}) u_{1} u_{2}\right) d S .
$$

An estimate of $I$ is needed with respect to the norm $\|y\|_{2}+\|u\|_{L^{2}(\Gamma)}$ (cf. (4.19)). We therefore have to require at least $\bar{\varphi} \in L^{2}(\Gamma)$ in the second item and $\bar{\varphi} \in L^{\infty}(\Gamma)$ in the third one. On the other hand, only $\bar{\varphi} \in L^{r}(\Gamma)$ follows from $\bar{\varphi} \in W^{1, \sigma}(\Omega)$ for $r<(n-1) /(n-2)$; see Nečas [17, p. 84]. For $n=2$ we obtain $\bar{\varphi} \in L^{r}(\Gamma)$ for all $r<\infty$, while $n=3$ yields the regularity $\bar{\varphi} \in L^{r}(\Gamma)$ for all $r<2$. On account of this, the following additional assumption is crucial for our analysis.

(A4) Let one of the following statements be true:

(i) $\bar{\varphi} \in L^{\infty}(\Gamma)$.

(ii) $b_{u u}(x, y, u)=0$ on $\Gamma \times \mathbb{R}^{2}$ and, if $n \geq 3$, then $\bar{\varphi} \in L^{r}(\Gamma)$ for some $r>n-1$.

(iii) $b_{u u}(x, y, u)=b_{y u}(x, y, u)=0$ on $\Gamma \times \mathbb{R}^{2}$ and, if $n \geq 4$, then $\bar{\varphi} \in L^{r}(\Gamma)$ for some $r>(n-1) / 2$.

(iv) $b^{\prime \prime}(\cdot, y, u)=0$.

We briefly comment on the consequences of these assumptions: (i) is true if $\bar{f}_{y} \in L^{q}(\Omega), \bar{g}_{y} \in L^{p}(\Gamma)$ and if the restrictions of $F_{i}^{\prime}, i=1, \ldots, m$, and $E^{\prime}(\bar{y})^{*} \bar{z}^{*}$ to $\Omega$ and $\Gamma$, respectively, belong to $L^{q}(\Omega), L^{p}(\Gamma)$, as well. Moreover, (i) holds for functionals $F_{i}^{\prime}, i=1, \ldots, m$, and $E^{\prime}(\bar{y})^{*} \bar{z}^{*}$ of $C(\bar{\Omega})^{*}$, where the associated real Borel measures are concentrated on the set $A \subset \Omega$.

In addition to some assumptions on the regularity of $\bar{\varphi}$ for $n \geq 3,4$, (ii) requires linearity of $b$ with respect to $u$, that is $b(x, y, u)=b_{0}(x, y)+b_{1}(x, y) u$, (iii) means that $b(x, y, u)=b_{1}(x, y)+b_{2}(x) u$, while (iv) is true only for an affine-linear boundary condition (but yet also true for a nonlinear functional $F_{0}$ ).

(A4) is obviously satisfied in the example (P).

As a consequence of (A3) and (A4), pointwise state-constraints on the whole set $\bar{\Omega}$ can only be handled by the standard part of our theory if $u$ appears linearly in the boundary condition and $n=2$. In the considerations below, we denote by $r_{i}^{T}$ the remainder terms associated with the $i$ th order Taylor expansion of a mapping $T$. For instance, the following first and second order expansions of $b(x, y, u)$ are used at triplets $(x, y, u)$ and $(x, \bar{y}, \bar{u}) \in \mathbb{R}^{n+2}$ :

$$
b(x, y, u)-b(x, \bar{y}, \bar{u})=b^{\prime}(x, \bar{y}, \bar{u})(y-\bar{y}, u-\bar{u})+r_{1}^{b},
$$

where

$$
r_{1}^{b}=\left(b_{y}^{\vartheta}-\bar{b}_{y}\right)(y-\bar{y})+\left(b_{u}^{\vartheta}-\bar{b}_{u}\right)(u-\bar{u})
$$

and $b_{y}^{\vartheta}, b_{u}^{\vartheta}, \bar{b}_{y}, \bar{b}_{u}$ denote $b_{y}, b_{u}$ taken at $(x, \bar{y}+\vartheta(y-\bar{y}), \bar{u}+\vartheta(u-\bar{u}))$ and $(x, \bar{y}, \bar{u})$, respectively, with some $\vartheta \in(0,1)$. Expanding the same expression up to the order two, we have

$$
\begin{aligned}
b(x, y, u)-b(x, \bar{y}, \bar{u})= & b^{\prime}(x, \bar{y}, \bar{u})(y-\bar{y}, u-\bar{u}) \\
& +\frac{1}{2}(y-\bar{y}, u-\bar{u}) b^{\prime \prime}(x, \bar{y}, \bar{u})\left(\begin{array}{c}
y-\bar{y} \\
u-\bar{u}
\end{array}\right)+r_{2}^{b},
\end{aligned}
$$


with the second order remainder term

$$
r_{2}^{b}=\frac{1}{2}(y-\bar{y}, u-\bar{u})\left[b^{\prime \prime, \vartheta}-\bar{b}^{\prime \prime}\right](y-\bar{y}, u-\bar{u})^{T} .
$$

Here, $b^{\prime \prime}, \vartheta, \bar{b}^{\prime \prime}$ denote the Hessian matrix of $b$ with respect to $(y, u)$ taken at the same triplets as above. Due to our assumptions on $b^{\prime}$ and $b^{\prime \prime}$, the estimates

$$
\begin{aligned}
& \left|r_{1}^{b}\right| \leq C_{M}\left(|y-\bar{y}|^{2}+|u-\bar{u}|^{2}\right), \\
& \left|r_{2}^{b}\right| \leq C_{M} \eta(|y-\bar{y}|+|u-\bar{u}|)\left(|y-\bar{y}|^{2}+|u-\bar{u}|^{2}\right)
\end{aligned}
$$

are valid $\forall|y|,|\bar{y}|,|u|,|\bar{u}| \leq M$. We continue with the discussion of the remainders $r_{1}^{\mathcal{L}}$ and $r_{2}^{\mathcal{L}}$. A Taylor expansion of $\mathcal{L}$ gives

$$
\begin{aligned}
& \mathcal{L}\left(y, u, \bar{\varphi}, \bar{\lambda}, \bar{z}^{*}\right)-\mathcal{L}\left(\bar{y}, \bar{u}, \bar{\varphi}, \bar{\lambda}, \bar{z}^{*}\right) \\
= & \overline{\mathcal{L}}_{y}(y-\bar{y})+\overline{\mathcal{L}}_{u}(u-\bar{u})+r_{1}^{\mathcal{L}} \\
= & \overline{\mathcal{L}}_{y}(y-\bar{y})+\overline{\mathcal{L}}_{u}(u-\bar{u})+\frac{1}{2}\left(\overline{\mathcal{L}}_{y y}[y-\bar{y}]^{2}+2 \overline{\mathcal{L}}_{y u}[y-\bar{y}, u-\bar{u}]+\overline{\mathcal{L}}_{u u}[u-\bar{u}]^{2}\right)+r_{2}^{\mathcal{L}},
\end{aligned}
$$

where $\overline{\mathcal{L}}$ indicates that $\mathcal{L}$ and its derivatives are taken at $\left(\bar{y}, \bar{u}, \bar{\varphi}, \bar{\lambda}, \bar{z}^{*}\right)$. We have

$$
\begin{aligned}
& r_{1}^{\mathcal{L}}=\left(\mathcal{L}_{y}^{\vartheta}-\overline{\mathcal{L}}_{y}\right)(y-\bar{y})+\left(\mathcal{L}_{u}^{\vartheta}-\overline{\mathcal{L}}_{u}\right)(u-\bar{u}) \\
& r_{2}^{\mathcal{L}}=\frac{1}{2}\left(\left(\mathcal{L}_{y y}^{\vartheta}-\overline{\mathcal{L}}_{y y}\right)[y-\bar{y}]^{2}+2\left(\mathcal{L}_{y u}^{\vartheta}-\overline{\mathcal{L}}_{y u}\right)[y-\bar{y}, u-\bar{u}]+\left(\mathcal{L}_{u u}^{\vartheta}-\overline{\mathcal{L}}_{u u}\right)[u-\bar{u}]^{2}\right) .
\end{aligned}
$$

$\mathcal{L}^{\vartheta}$ indicates that $\left(\bar{y}+\vartheta(y-\bar{y}), \bar{u}+\vartheta(u-\bar{u}), \bar{\varphi}, \bar{\lambda}, \bar{z}^{*}\right)$ is substituted for $\left(y, u, \bar{\varphi}, \bar{\lambda}, \bar{z}^{*}\right)$ in $\mathcal{L}^{\prime}$ and $\mathcal{L}^{\prime \prime}$ with some $\vartheta \in(0,1)$. On account of the assumptions (A1)-(A4), we are able to verify

$$
\begin{aligned}
& \left|r_{1}^{\mathcal{L}}\right| \leq C_{\mathcal{L}}\left(\|y-\bar{y}\|_{2}^{2}+\|u-\bar{u}\|_{L^{2}(\Gamma)}^{2}\right) \\
& \left|r_{2}^{\mathcal{L}}\right| \leq C_{\mathcal{L}} \eta\left(\|y-\bar{y}\|_{C(\bar{\Omega})}+\|u-\bar{u}\|_{L^{\infty}(\Gamma)}\right) \cdot\left(\|y-\bar{y}\|_{2}^{2}+\|u-\bar{u}\|_{L^{2}(\Gamma)}^{2}\right)
\end{aligned}
$$

and

$$
\left|\overline{\mathcal{L}}^{\prime \prime}\left[\left(y_{1}, u_{1}\right),\left(y_{2}, u_{2}\right)\right]\right| \leq C_{\mathcal{L}}\left(\left\|y_{1}\right\|_{2}+\left\|u_{1}\right\|_{L^{2}(\Gamma)}\right)\left(\left\|y_{2}\right\|_{2}+\left\|u_{2}\right\|_{L^{2}(\Gamma)}\right) .
$$

The constant $C_{\mathcal{L}}>0$ depends in particular on $\bar{\varphi}$. For the definition of $\eta$ we refer to the assumption (A2). The analysis of (4.17)-(4.19) is performed in Appendix 7.3.

5. Standard second order sufficient optimality condition. Our main aim is to establish sufficient optimality conditions close to the necessary ones derived in Casas and Tröltzsch [8]. Therefore, we include also certain first order sufficient optimality conditions. We shall combine an approach going back to Zowe and Maurer [15] with a splitting technique introduced by Dontchev et al. [10]. The method of [10] was focused on the optimal control of ordinary differential equations. It was extended later by the authors in [9] to the case of elliptic equations without state-constraints.

In [15], Maurer and Zowe introduced first order sufficient optimality conditions for differentiable optimization problems subject to a general constraint $g(w) \leq 0$. For our problem, the application of their approach in its full generality is rather technical. Therefore, in an initial step we incorporate the first order sufficient optimality condition only for the constraints on the control. Later, we shall deal in the same way with additional state-constraints. 
The role of first order sufficient conditions can be explained most easily by the minimization problem $\left\{\min f(x) \mid x_{a} \leq x \leq x_{b}\right\}$, where $f: \mathbb{R}^{n} \rightarrow \mathbb{R}$ is of class $C^{2}$. Let $\bar{x}$ satisfy the first order necessary conditions (variational inequality). If $n=1$, then $f^{\prime}(\bar{x}) \neq 0$ implies that $\bar{x}$ is a local minimizer (even for concave $f$ ). Therefore, the second order sufficient optimality condition $f^{\prime \prime}(\bar{x})>0$ is needed only in the case $f^{\prime}(\bar{x})=0$, where the first order necessary condition is not sufficient. The situation is similar for $n>1$ : The positive definiteness of $f^{\prime \prime}(\bar{x})$ has to be required only on the subspace $\left\{x \in \mathbb{R}^{n} \mid x^{i}=0\right.$ if $\left.D^{i} f(\bar{x}) \neq 0\right\}$.

Define for fixed $\tau>0$ (arbitrarily small) the set

$$
\Gamma_{\tau}=\left\{x \in \Gamma|| g_{u}(x, \bar{y}(x), \bar{u}(x))+\bar{\varphi}(x) b_{u}(x, \bar{y}(x), \bar{u}(x)) \mid \geq \tau\right\} .
$$

$\Gamma_{\tau}$ is a subset of "strongly active" control constraints (cf. (3.5)). In other words, $\Gamma_{\tau}=$ $\left\{x \in \Gamma|\quad| \mathcal{L}_{u}\left(\bar{y}, \bar{u}, \bar{\varphi}, \bar{\lambda}, \bar{z}^{*}\right)(x) \mid \geq \tau\right\}$ is the set, where the gradient of the objective (expressed as a function of the control) is sufficiently steep. In the example above, $\tau$ can be chosen as the minimal value of all nonvanishing $\left|D^{i} f(\bar{x})\right|$.

We mention at this point the relation

$$
\left\langle\bar{z}^{*}, E^{\prime}(\bar{y}) y\right\rangle \leq 0
$$

$\forall(y, u) \in L(\mathcal{M}, \bar{w})$, which follows from $\left\langle\bar{z}^{*}, E^{\prime}(\bar{y}) y\right\rangle=\varrho\left\langle\bar{z}^{*}, \kappa-E(\bar{y})\right\rangle \leq 0$ in view of (3.4).

Let $\mathcal{P}_{\tau}: L^{\infty}(\Gamma) \rightarrow L^{\infty}(\Gamma)$ denote the projection operator $u \mapsto \chi_{\Gamma \backslash \Gamma_{\tau}} u=\mathcal{P}_{\tau} u$. In other words, $\left(\mathcal{P}_{\tau} u\right)(x)=u(x)$ holds on $\Gamma \backslash \Gamma_{\tau}$, while $\left(\mathcal{P}_{\tau} u\right)(x)=0$ holds on $\Gamma_{\tau}$. We begin with our first and at the same time simplest second order sufficient optimality condition.

(SSC) There exist positive numbers $\tau$ and $\delta$ such that

$$
\mathcal{L}^{\prime \prime}\left(\bar{y}, \bar{u}, \bar{\varphi}, \bar{\lambda}, \bar{z}^{*}\right)\left[w_{2}, w_{2}\right] \geq \delta\left\|u_{2}\right\|_{L^{2}(\Gamma)}^{2}
$$

holds for all pairs $w_{2}=\left(y_{2}, u_{2}\right)$ constructed in the following way: For every $w=(y, u) \in L(\mathcal{M}, \bar{w})$, we split up the control part $u$ in $u_{1}=\left(u-\mathcal{P}_{\tau} u\right)$ and $u_{2}=\mathcal{P}_{\tau} u$. The solutions of the linearized state equation

$$
\left\{\begin{aligned}
-\Delta y_{i}+y_{i} & =0 & & \text { in } \Omega, \\
\partial_{\nu} y_{i} & =b_{y}(\cdot, \bar{y}, \bar{u}) y_{i}+b_{u}(\cdot, \bar{y}, \bar{u}) u_{i} & & \text { on } \Gamma
\end{aligned}\right.
$$

associated with $u_{i}$ are denoted by $y_{i}, i=1,2$. By this construction, we get the representation $w=w_{1}+w_{2}=\left(y_{1}, u_{1}\right)+\left(y_{2}, u_{2}\right)$.

REMARK 5.1. The coercitivity condition (5.2) of (SSC) is required on the whole set $L(\mathcal{M}, \bar{w})$ if $\Gamma_{\tau}$ is empty. This rather strong second order condition is obtained by the formal setting $\tau=\infty$.

THEOREM 5.2. Let the feasible pair $\bar{w}=(\bar{y}, \bar{u})$ satisfy the regularity condition (R), the first order necessary optimality conditions (3.9)-(3.11), and the second order sufficient optimality condition (SSC). Suppose further that the general assumptions (A1)-(A4) are satisfied. Then there are constants $\varrho>0$ and $\delta^{\prime}>0$ such that

$$
F_{0}(\hat{y}, \hat{u}) \geq F_{0}(\bar{y}, \bar{u})+\delta^{\prime}\|\hat{u}-\bar{u}\|_{L^{2}(\Gamma)}^{2}
$$

holds for all feasible pairs $\hat{w}=(\hat{y}, \hat{u})$ such that

$$
\|\hat{u}-\bar{u}\|_{L^{\infty}(\Gamma)}<\varrho .
$$


Proof. We denote by $\bar{l}=\left(\bar{\varphi}, \bar{\lambda}, \bar{z}^{*}\right)$ the triplet of Lagrange multipliers appearing in the first order necessary optimality conditions. Let an arbitrary feasible pair $\hat{w}=$ $(\hat{y}, \hat{u})$ be given. Then

$$
F_{0}(\hat{w})-F_{0}(\bar{w})=\mathcal{L}(\hat{w}, \bar{l})-\mathcal{L}(\bar{w}, \bar{l})-\left\langle\bar{z}^{*}, E(\hat{y})-E(\bar{y})\right\rangle
$$

follows from $F(\hat{w})=F(\bar{w})=0$. The complementary slackness condition implies

$$
\left.-\left\langle\bar{z}^{*}, E(\hat{y})-E(\bar{y})\right)\right\rangle \geq 0 .
$$

Hence we can neglect this term, and a second order Taylor expansion yields

$$
\begin{aligned}
F_{0}(\hat{w})-F_{0}(\bar{w}) & \geq \mathcal{L}(\hat{w}, \bar{l})-\mathcal{L}(\bar{w}, \bar{l}) \\
& \geq \int_{\Gamma} l_{u}(\hat{u}-\bar{u}) d S+\frac{1}{2} \mathcal{L}^{\prime \prime}(\bar{w}, \bar{l})[\hat{w}-\bar{w}]^{2}+r_{2}^{\mathcal{L}}(\bar{w}, \hat{w}-\bar{w}),
\end{aligned}
$$

where $l_{u}(x)=g_{u}(x, \bar{y}(x), \bar{u}(x))+\bar{\varphi}(x) b_{u}(x, \bar{y}(x), \bar{u}(x))$. Using the variational inequality, we find

$$
F_{0}(\hat{w})-F(\bar{w}) \geq \tau \int_{\Gamma_{\tau}}|\hat{u}-\bar{u}| d S+\frac{1}{2} \mathcal{L}^{\prime \prime}(\bar{w}, \bar{l})[\hat{w}-\bar{w}]^{2}+r_{2}^{\mathcal{L}}(\bar{w}, \hat{w}-\bar{w}) .
$$

Let us introduce for convenience the bilinear form $B=\mathcal{L}^{\prime \prime}(\bar{w}, \bar{l})$. Next we approximate $\hat{w}-\bar{w}$ by $w=(y, u) \in L(\mathcal{M}, \bar{w})$, according to Theorem 4.2. In this way we get a remainder $r=\left(r^{y}, r^{u}\right)=\hat{w}-\bar{w}-w$ satisfying the estimate

$$
\|r\| \leq C_{L}\|\hat{u}-\bar{u}\|_{L^{\infty}(\Gamma)}\|\hat{u}-\bar{u}\|_{L^{2}(\Gamma)} .
$$

It follows that $B[\hat{w}-\bar{w}]^{2}=B[w]^{2}+2 B[r, w]+B[r]^{2}$. We have $w \in L(\mathcal{M}, \bar{w})$; hence (SSC) applies to $B[w]^{2}$. Now we substitute in $B[w]^{2}$ the representation $w=w_{1}+w_{2}$ described in (SSC) and deduce

$$
\begin{aligned}
B[w]^{2}= & B\left[w_{2}\right]^{2}+2 B\left[w_{1}, w_{2}\right]+B\left[w_{1}\right]^{2} \\
\geq & \delta\left\|u_{2}\right\|_{L^{2}(\Gamma)}^{2}-2 C_{\mathcal{L}}\left(\left\|y_{1}\right\|_{2}+\left\|u_{1}\right\|_{L^{2}(\Gamma)}\right)\left(\left\|y_{2}\right\|_{2}+\left\|u_{2}\right\|_{L^{2}(\Gamma)}\right) \\
& -C_{\mathcal{L}}\left(\left\|y_{1}\right\|_{2}+\left\|u_{1}\right\|_{L^{2}(\Gamma)}\right)^{2}
\end{aligned}
$$

from (SSC) and (4.19). In the following, $c$ will denote a generic constant. Suppose that $\varrho<1$ is given and assume $\|\hat{u}-\bar{u}\|_{L^{\infty}(\Gamma)}<\varrho$. Then $\left\|y_{i}\right\|_{2} \leq c\left\|u_{i}\right\|_{L^{2}(\Gamma)}$ and Young's inequality together yield

$$
\begin{aligned}
B[w]^{2} \geq & \delta\left\|u_{2}\right\|_{L^{2}(\Gamma)}^{2}-\frac{\delta}{2}\left\|u_{2}\right\|_{L^{2}(\Gamma)}^{2}-c\left\|u_{1}\right\|_{L^{2}(\Gamma)}^{2} \\
\geq & \frac{\delta}{2} \int_{\Gamma \backslash \Gamma_{\tau}} u^{2} d S-c \int_{\Gamma_{\tau}} u^{2} d S \\
\geq & \frac{\delta}{2} \int_{\Gamma_{\curlyvee} \Gamma_{\tau}}|\hat{u}-\bar{u}|^{2} d S-c \int_{\Gamma \backslash \Gamma_{\tau}}|\hat{u}-\bar{u}|\left|r^{u}\right| d S-c \int_{\Gamma_{\tau}}|\hat{u}-\bar{u}|^{2} d S \\
& -c \int_{\Gamma_{\tau}}|\hat{u}-\bar{u}|\left|r^{u}\right| d S-c \int_{\Gamma_{\tau}}\left|r^{u}\right|^{2} d S .
\end{aligned}
$$

The expression under the third integral is estimated by $\|\hat{u}-\bar{u}\|_{L^{\infty}(\Gamma)}|\hat{u}-\bar{u}|$. In the other integrals (except the first) we insert (5.8) and derive

$$
B[w]^{2} \geq \frac{\delta}{2} \int_{\Gamma \backslash \Gamma_{\tau}}|\hat{u}-\bar{u}|^{2} d S-c \varrho \int_{\Gamma_{\tau}}|\hat{u}-\bar{u}| d S-c \varrho\|\hat{u}-\bar{u}\|_{L^{2}(\Gamma)}^{2} .
$$


The treatment of $B[r, w]$ and $B[r]^{2}$ is simpler. We find

$$
\begin{aligned}
|B[r, w]| & \leq c\|r\|\|u\|_{L^{2}(\Gamma)}=c\|r\|\left\|\hat{u}-\bar{u}+r^{u}\right\|_{L^{2}(\Gamma)} \\
& \leq c \varrho\|\hat{u}-\bar{u}\|_{L^{2}(\Gamma)}^{2} .
\end{aligned}
$$

The same type of estimate applies to $B[r]^{2}$. Altogether,

$$
B[\hat{w}-\bar{w}]^{2} \geq \frac{\delta}{2} \int_{\Gamma \backslash \Gamma_{\tau}}|\hat{u}-\bar{u}|^{2} d S-c \varrho \int_{\Gamma_{\tau}}|\hat{u}-\bar{u}| d S-c \varrho\|\hat{u}-\bar{u}\|_{L^{2}(\Gamma)}^{2}
$$

is obtained. By substituting (5.11) in (5.7), we get

$$
\begin{aligned}
F_{0}(\hat{w})-F_{0}(\bar{w}) \geq & (\tau-c \varrho) \int_{\Gamma_{\tau}}|\hat{u}-\bar{u}| d S+\frac{\delta}{2} \int_{\Gamma \backslash \Gamma_{\tau}}|\hat{u}-\bar{u}|^{2} d S-c \varrho\|\hat{u}-\bar{u}\|_{L^{2}(\Gamma)}^{2} \\
& -\left|r_{2}^{\mathcal{L}}(\bar{w}, \hat{w}-\bar{w})\right| \\
\geq & \frac{\tau}{2} \int_{\Gamma_{\tau}}|\hat{u}-\bar{u}| d S+\frac{\delta}{2} \int_{\Gamma \backslash \Gamma_{\tau}}|\hat{u}-\bar{u}|^{2} d S-c \varrho\|\hat{u}-\bar{u}\|_{L^{2}(\Gamma)}^{2} \\
& -\left|r_{2}^{\mathcal{L}}(\bar{w}, \hat{w}-\bar{w})\right| .
\end{aligned}
$$

Since $\|\hat{u}-\bar{u}\|_{L^{\infty}(\Gamma)} \leq 1$ was assumed, $|\hat{u}-\bar{u}| \geq|\hat{u}-\bar{u}|^{2}$ holds a.e. Using this in the first integral, setting $\delta^{\prime}=\min \{\tau / 2, \delta / 2\}$, and substituting the estimate (4.18) for $r_{2}^{\mathcal{L}}$, we complete our estimation by

$$
\begin{aligned}
F_{0}(\hat{w})-F_{0}(\bar{w}) & \geq\|\hat{u}-\bar{u}\|_{L^{2}(\Gamma)}^{2}\left(\delta^{\prime}-c \varrho-\eta\left(c\|\hat{u}-\bar{u}\|_{L^{\infty}(\Gamma)}\right)\right) \\
& \geq \frac{\delta^{\prime}}{2}\|\hat{u}-\bar{u}\|_{L^{2}(\Gamma)}^{2}
\end{aligned}
$$

for sufficiently small $\varrho>0$.

Our condition (SSC) does not have the form expected from a comparison with second order conditions in finite dimensional spaces. In particular, the pair $\left(y_{2}, u_{2}\right)$ constructed in (SSC) does not in general belong to $L(\mathcal{M}, \bar{w})$. To overcome this difficulty, we introduce another regularity condition $(R)_{\tau}$ that is stronger than $(R)$. This new constraint qualification is similar to that one used in Casas and Tröltzsch [8] to derive second order necessary conditions.

Let $\mathcal{C}_{\tau}(\bar{u})$ denote the set of controls $u \in \mathcal{C}(\bar{u})$ having the property $u(x)=0$ if $x \in \Gamma_{\tau}$. We strengthen (R) to

$$
T^{\prime}(\bar{y}) G^{\prime}(\bar{u}) \mathcal{C}_{\tau}(\bar{u})-\boldsymbol{K}(T(\bar{y}))=\boldsymbol{Z} .
$$

On using $(\mathrm{R})_{\tau}$, we are able to show that the following second order sufficient optimality condition implies (5.4) as well.

$(\mathrm{SSC})_{\tau}$ There exist positive numbers $\tau$ and $\delta$ such that

$$
\mathcal{L}^{\prime \prime}\left(\bar{y}, \bar{u}, \bar{\varphi}, \bar{\lambda}, \bar{z}^{*}\right)[w, w] \geq \delta\|u\|_{L^{2}(\Gamma)}^{2}
$$

holds for all pairs $w=(y, u)$ of $L(\mathcal{M}, \bar{w})$ with the property $u(x)=0$ for almost every $x \in \Gamma_{\tau}$.

THEOREM 5.3. Let the assumptions of Theorem 5.2 be fulfilled, where (R) and $(\mathrm{SSC})$ are replaced by $(\mathrm{R})_{\tau}$ and $(\mathrm{SSC})_{\tau}$. Then the assertion of Theorem 5.2 remains true. 
Proof. The proof is almost identical to that of Theorem 5.2. The only difference consists in a more detailed splitting. In the first part of the proof we repeat the steps up to the splitting $w=w_{1}+w_{2}$ after (5.8). Define $\Phi=T \circ G$. Then we have

$$
\Phi^{\prime}(\bar{u})\left(u_{1}+u_{2}\right) \in K(\Phi(\bar{u}))
$$

as $w_{1}+w_{2} \in L(\mathcal{M}, \bar{w})$. Therefore,

$$
\Phi^{\prime}(\bar{u}) u_{2} \in K(\Phi(\bar{u}))-\Phi^{\prime}(\bar{u}) u_{1}
$$

holds so that $w_{2}=\left(y_{2}, u_{2}\right)$ does not in general belong to the linearized cone. Thanks to the regularity condition $(\mathrm{R})_{\tau}$, the linear version of the Robinson-Ursescu theorem (see Robinson [18]) implies the existence of $u_{H}$ in $\mathcal{C}_{\tau}(\bar{u})$ with the following properties: The inclusion

$$
\Phi^{\prime}(\bar{u}) u_{H} \in K(\Phi(\bar{u}))
$$

holds, and

$$
\left\|u_{2}-u_{H}\right\|_{L^{2}(\Gamma)} \leq c\left\|u_{1}\right\|_{L^{2}(\Gamma)}
$$

is satisfied (see the proof of Theorem 4.2 in the appendix). In other words, we find a pair $w_{H}=\left(y_{H}, u_{H}\right)$ in $L(\mathcal{M}, \bar{w})$ with $u_{H}=0$ on $\Gamma_{\tau}$. Hence, (SSC) applies to $B\left[w_{H}\right]^{2}$. Moreover, the control $u_{H}$ is sufficiently close to $u_{2}$.

Now we define $\tilde{u}_{2}=u_{H}$ and $\tilde{u}_{1}=u_{1}+\left(u_{2}-u_{H}\right)$. Further, let $\tilde{y}_{i}=G^{\prime}(\bar{u}) \tilde{u}_{i}$ denote the corresponding solution of the linearized state equation. Then $\tilde{w}_{i}=\left(\tilde{y}_{i}, \tilde{u}_{i}\right)$ is substituted for $w_{i}=\left(y_{i}, u_{i}\right), i=1,2$. The only difference between the proofs of Theorem 5.2 and 5.3 appears between the formulas and (5.8) and (5.9): We use the splitting $w=\tilde{w}_{1}+\tilde{w}_{2}$ instead of $w=w_{1}+w_{2}$. Moreover, the first line of the estimate (5.9) is changed as follows:

$$
\begin{aligned}
B[w]^{2} & \geq \delta\left\|\tilde{u}_{2}\right\|_{L^{2}(\Gamma)}^{2}-\frac{\delta}{4}\left\|\tilde{u}_{2}\right\|_{L^{2}(\Gamma)}^{2}-c\left\|\tilde{u}_{1}\right\|_{L^{2}(\Gamma)}^{2} \\
& \geq \frac{3 \delta}{4}\left\|u_{2}+\left(u_{H}-u_{2}\right)\right\|_{L^{2}(\Gamma)}^{2}-c\left\|u_{1}+\left(u_{2}-u_{H}\right)\right\|_{L^{2}(\Gamma)}^{2} \\
& \geq \frac{\delta}{2}\left\|u_{2}\right\|_{L^{2}(\Gamma)}^{2}-c\left\|u_{1}\right\|_{L^{2}(\Gamma)}^{2},
\end{aligned}
$$

where we have used the estimate (5.13). Then we proceed word for word as in the proof of Theorem 5.2.

Example. Let us briefly comment on (SSC) in the case of $(\mathrm{P})$ for an active state constraint $\bar{y}(0)=y_{0}$. Then $L(\mathcal{M}, \bar{w})$ is expressed through (4.4), (4.5), and a quite strong second order condition is formulated by

$$
\overline{\mathcal{L}}^{\prime \prime}\left(\bar{y}, \bar{u}, \bar{\varphi}, \bar{z}^{*}\right)[w, w] \geq \delta\|u\|_{L^{2}(\Gamma)}^{2}
$$

for all $w=(y, u) \in L(\mathcal{M}, \bar{w})$. In this way, we would not take advantage of strongly active control constraints. These constraints appear on $\Gamma_{\tau}=\{x \in \Gamma|| \alpha \bar{u}(x)+\bar{\varphi}(x) \mid \geq$ $\tau \mid\}$. We split $(y, u)=\left(y_{1}, u_{1}\right)+\left(y_{2}, u_{2}\right)$, where $u_{2}=0$ on $\Gamma_{\tau}$ and $u_{1}=0$ on $\Gamma \backslash \Gamma_{\tau}$. (SSC) requires the coercitivity condition (5.14) only for $\left(y_{2}, u_{2}\right)$, while $\left(y_{1}, u_{1}\right)$ is handled by first order sufficient optimality conditions. Notice that $y_{2}$ might violate the state-constraint $y\left(x_{0}\right) \leq 0$. We avoid this by $(\mathrm{SSC})_{\tau}$ : It requires the coercitivity 
condition for the following $u \in \mathcal{C}(\bar{u})$ : They vanish on $\Gamma_{\tau}$ and satisfy together with the associated solution $y$ of the linearized partial differential equation the state-constraint $y\left(x_{0}\right) \leq 0$.

The paper [15] shows that "strongly active" state-constraints may also contribute terms to the first order sufficient optimality conditions. However, this leads to a rather technical construction and more restrictive assumptions. We have to suppose that the function $b$ is linear with respect to the control $u$ and $n=2$. The corresponding theorem is stated below. Define for fixed $\beta>0$ and $\tau>0$ the following subset of $L(\mathcal{M}, \bar{w})$ :

$$
L_{\beta, \tau}(\mathcal{M}, \bar{w})=\{w \mid w=(y, u) \in L(\mathcal{M}, \bar{w}) \text { and } w \text { satisfies (5.15) below }\} .
$$

The decisive inequality characterizing $L_{\beta, \tau}$ is

$$
\left\langle\bar{z}^{*}, E^{\prime}(\bar{y}) y\right\rangle \geq-\beta \int_{\Gamma \backslash \Gamma_{\tau}}|u(x)| d S(x) .
$$

$L_{\beta, \tau}(\mathcal{M}, \bar{w})$ is the subset of $L(\mathcal{M}, \bar{w})$, where the term $\left\langle\bar{z}^{*}, E(y)\right\rangle$ does not much contribute to the first order sufficient optimality condition. It is only this set $L_{\beta, \tau}(\mathcal{M}, \bar{w})$ where we have to require second order conditions, namely, the following condition.

$\left(\mathrm{SSC}^{\prime}\right)$ There exist positive numbers $\beta, \tau$, and $\delta$ such that

$$
\mathcal{L}^{\prime \prime}\left(\bar{y}, \bar{u}, \bar{\varphi}, \bar{\lambda}, \bar{z}^{*}\right)\left[w_{2}, w_{2}\right] \geq \delta\left\|u_{2}\right\|_{L^{2}(\Gamma)}^{2}
$$

holds for all $w_{2}=\left(y_{2}, u_{2}\right)$ obtained in the same way introduced in (SSC) by elements $w$ taken from the smaller set $L_{\beta, \tau}(\mathcal{M}, \bar{w})$.

Using this condition, we formulate the following.

THEOREM 5.4. Let the feasible pair $\bar{w}=(\bar{y}, \bar{u})$ satisfy the regularity condition $(\mathrm{R})$, the first order necessary optimality conditions (3.9)-(3.11), and the second order sufficient optimality condition ( $\left.\mathrm{SSC}^{\prime}\right)$. Suppose further that the general assumptions (A1)-(A4) are satisfied. Moreover, assume that $n=2$ and $b(x, y, u)=b_{1}(x, y)+$ $b_{2}(x) u$. Then there are constants $\varrho>0$ and $\delta^{\prime}>0$ such that

$$
F_{0}(\hat{y}, \hat{u}) \geq F_{0}(\bar{y}, \bar{u})+\delta^{\prime}\|\hat{u}-\bar{u}\|_{L^{2}(\Gamma)}^{2}
$$

holds for all feasible pairs $\hat{w}=(\hat{y}, \hat{u})$ satisfying

$$
\|\hat{u}-\bar{u}\|_{L^{\infty}(\Gamma)}<\varrho .
$$

Proof. We begin in the way we have shown Theorem 5.2 by

$$
F_{0}(\hat{w})-F_{0}(\bar{w})=\mathcal{L}(\hat{w}, \bar{l})-\mathcal{L}(\bar{w}, \bar{l})-\left\langle\bar{z}^{*}, E(\hat{y})-E(\bar{y})\right\rangle .
$$

Once again, the representation $\hat{w}-\bar{w}=w+r$ is obtained. Now we distinguish between two cases.

Case I: $w=(y, u) \in L(\mathcal{M}, \bar{w}) \backslash L_{\beta, \tau}(\mathcal{M}, \bar{w})$. This is the case where we deduce (5.17) from first order sufficiency. Here, the inequality

$$
-\left\langle\bar{z}^{*}, E^{\prime}(\bar{y}) y\right\rangle>\beta \int_{\Gamma \backslash \Gamma_{\tau}}|u(x)| d S(x)
$$


is fulfilled. We transform (5.19) as follows:

$$
\begin{aligned}
F_{0}(\hat{w})-F_{0}(\bar{w})= & \mathcal{L}^{\prime}(\bar{w}, \bar{l})(\hat{w}-\bar{w})+r_{1}^{\mathcal{L}}(\bar{w}, \hat{w}-\bar{w})-\left\langle\bar{z}^{*}, E(\hat{y})-E(\bar{y})\right\rangle \\
= & \mathcal{L}_{y}(\bar{w}, \bar{l})(\hat{y}-\bar{y})+\mathcal{L}_{u}(\bar{w}, \bar{l})(\hat{u}-\bar{u})-\left\langle\bar{z}^{*}, E^{\prime}(\bar{y})(\hat{y}-\bar{y})\right\rangle \\
& +r_{1}^{\mathcal{L}}(\bar{w}, \hat{w}-\bar{w})-\left\langle\bar{z}^{*}, r_{1}^{E}(\bar{y}, \hat{y}-\bar{y})\right\rangle \\
= & 0+\int_{\Gamma} l_{u}(x)(\hat{u}(x)-\bar{u}(x)) d S(x)-\left\langle\bar{z}^{*}, E^{\prime}(\bar{y}) y\right\rangle \\
& +r_{1}^{\mathcal{L}}(\bar{w}, \hat{w}-\bar{w})-\left\langle\bar{z}^{*}, E^{\prime}(\bar{y}) r^{y}+r_{1}^{E}(\bar{y}, \hat{y}-\bar{y})\right\rangle,
\end{aligned}
$$

where $l_{u}(x)$ stands for $g_{u}(x, \bar{y}(x), \bar{u}(x))+\bar{\varphi}(x) b_{u}(x, \bar{y}(x), \bar{u}(x))$.

Owing to $n=2$ and $b(x, y, u)=b_{1}(x, y)+b_{2}(x) u$, we are able to apply the strong estimate (4.8) with $p=2$. This yields

$$
\|r\|_{Y \times L^{\infty}(\Gamma)} \leq C_{L, 2}\|\hat{u}-\bar{u}\|_{L^{2}(\Gamma)}^{2} .
$$

By Theorem 4.2, (5.22), (4.17), and (A3 (ii)) we have

$$
\max \left\{\left\|r^{y}\right\|_{2},\left|r_{1}^{\mathcal{L}}\right|,\left\|r_{1}^{E}\right\|_{Z}\right\} \leq c\left(\|\hat{y}-\bar{y}\|_{2}^{2}+\|\hat{u}-\bar{u}\|_{L^{2}(\Gamma)}^{2}\right) .
$$

Now the Lipschitz property of the mapping $u \mapsto y(u)=G(u)$ from $L^{2}(\Gamma)$ into $C(\bar{\Omega})$ (note that $n=2$ ) permits us to estimate the last three items of $(5.21)$ by $c\|\hat{u}-\bar{u}\|_{L^{2}(\Gamma)}^{2}$. (5.20) is applied to the second one, while the first one is treated by $\Gamma_{\tau}$ : We know that

$$
l_{u}(x)(\hat{u}(x)-\bar{u}(x)) \geq 0 \quad \text { a.e. on } \Gamma
$$

hence

$$
\int_{\Gamma} l_{u}(\hat{u}-\bar{u}) d S \geq \int_{\Gamma_{\tau}} l_{u}(\hat{u}-\bar{u}) d S=\int_{\Gamma_{\tau}}\left|l_{u}\right||\hat{u}-\bar{u}| d S \geq \tau \int_{\Gamma_{\tau}}|\hat{u}-\bar{u}| d S .
$$

Inserting this in (5.21) we continue with

$$
\begin{aligned}
F_{0}(\hat{w})-F_{0}(\bar{w}) & \geq \tau \int_{\Gamma_{\tau}}|\hat{u}-\bar{u}| d S+\beta \int_{\Gamma \backslash \Gamma_{\tau}}|u| d S-c\|\hat{u}-\bar{u}\|_{L^{2}(\Gamma)}^{2} \\
& \geq \tau \int_{\Gamma_{\tau}}|\hat{u}-\bar{u}| d S+\beta \int_{\Gamma \backslash \Gamma_{\tau}}|\hat{u}-\bar{u}| d S-c\|\hat{u}-\bar{u}\|_{L^{2}(\Gamma)}^{2}
\end{aligned}
$$

in view of $\left\|r^{u}\right\|_{L^{\infty}(\Gamma)} \leq c\|\hat{u}-\bar{u}\|_{L^{2}(\Gamma)}^{2}$. Proceeding with the estimation, we deduce

$$
\begin{aligned}
F_{0}(\hat{w})-F_{0}(\bar{w}) & \geq \min \{\beta, \tau\}\|\hat{u}-\bar{u}\|_{L^{1}(\Gamma)}-c \varrho\|\hat{u}-\bar{u}\|_{L^{1}(\Gamma)} \\
& \geq \beta^{\prime}\|\hat{u}-\bar{u}\|_{L^{1}(\Gamma)}
\end{aligned}
$$

with some $\beta^{\prime}>0$, provided that $\|\hat{u}-\bar{u}\|_{L^{\infty}(\Gamma)} \leq \varrho \leq \varrho_{1}$ is fulfilled and $\varrho_{1}$ is sufficiently small. Assume additionally that $\varrho_{1} \leq 1$. Then $|\hat{u}-\bar{u}|^{2} \leq|\hat{u}-\bar{u}|$ holds a.e.; hence

$$
F_{0}(\hat{w})-F_{0}(\bar{w}) \geq \beta^{\prime}\|\hat{u}-\bar{u}\|_{L^{2}(\Gamma)}^{2}
$$

follows for $\|\hat{u}-\bar{u}\|_{L^{\infty}(\Gamma)} \leq \varrho_{1}$.

Case II: $w \in L_{\beta, \tau}(\mathcal{M}, \bar{w})$ (partial use of first order sufficient optimality conditions). Here, we neglect the term $\left\langle\bar{z}^{*}, E(\hat{y})-E(\bar{y})\right\rangle$ and proceed word for word as in the proof of Theorem 5.2, using $L_{\beta, \tau}$ instead of $L$. 
REMARK 5.5. Unfortunately, the definition of $L_{\beta, \tau}(\mathcal{M}, \bar{w})$ is not constructive. It is difficult to describe in an explicit way which $(y, u) \in L(\mathcal{M}, \bar{w})$ belong to the different cases I or II. Therefore, this type of first order sufficient condition is only of limited value (see, for instance, the next example).

Example. To illustrate ( $\left.\mathrm{SSC}^{\prime}\right)$ for (P) in comparison with (SSC), let us assume for simplicity $\bar{u} \in \operatorname{int} \mathcal{U}^{a d}$, hence $\Gamma_{\tau}=\emptyset$. Then (SSC) requires the coercitivity condition (5.14) on the whole set $L(\mathcal{M}, \bar{w})$. If $\bar{y}(0)=y_{0}$ and $\bar{z}^{*}>0$ (strong complementarity), then $\left(\mathrm{SSC}^{\prime}\right)$ is weaker than (SSC): (5.14) is not needed for all $(y, u) \in L(\mathcal{M}, \bar{w})$ satisfying

$$
-\bar{z}^{*} y(0) \geq \beta \int_{\Gamma}|u(x)| d S .
$$

Assume that $y$ can be represented by a positive Green's function $G=G(x, \xi)$,

$$
y(0)=\int_{\Gamma} G(0, \xi) u(\xi) d S(\xi),
$$

such that $G(0, \xi) \geq \gamma>0$ on $\Gamma$. Then (5.24) is fulfilled with $\beta=\bar{z}^{*} \gamma \forall u \leq 0$. Moreover, all $u \geq 0, u \neq 0$ do not contribute to $L(\mathcal{M}, \bar{w})$. Therefore, the coercitivity condition (5.14) is needed only for all $u$ having positive and negative parts $U_{+}$and $U_{-}$, where $U_{+}$dominates $U_{-}$. However, this information does not essentially improve (SSC).

REMARK 5.6. Theorem 5.2 follows from Theorem 5.4 by setting $\beta=0$, where we can avoid the restrictions $n=2$ and $b(x, y, u)=b_{1}(x, y)+b_{2}(x) u$.

The cone $\mathcal{C}(\bar{u})$ is defined by $\mathcal{C}(\bar{u})=\left\{\rho(u-\bar{u}) \mid u \in \mathcal{U}^{\text {ad }}, \rho \geq 0\right\}$. Its closure in $L^{2}(\Gamma)$ is

$$
\operatorname{cl} \mathcal{C}(\bar{u})=\left\{v \in L^{2}(\Gamma) \mid v(x) \leq 0 \text { if } \bar{u}(x)=u_{b}(x), v(x) \geq 0, \text { if } \bar{u}(x)=u_{a}(x)\right\} .
$$

Let us redefine $L(\mathcal{M}, \bar{w})$ by substituting $\operatorname{cl} \mathcal{C}(\bar{u})$ for $\mathcal{C}(\bar{u})$ and require $(\mathrm{SSC})$ in this form. Then (SSC) appears to be stronger, and Theorem 5.2 holds as well, since $\operatorname{cl} \mathcal{C}(\bar{u}) \supset \mathcal{C}(\bar{u})$. However, it can be proved by $(\mathrm{R})$ and the generalized open mapping theorem that ( $\mathrm{SSC})$ based on $\mathrm{cl} \mathcal{C}(\bar{u})$ is in fact equivalent to (SSC) established with $\mathcal{C}(\bar{u})$. This follows by continuity arguments.

6. Extended second order conditions. A study of the preceding sections reveals that (SSC) is sufficient for local optimality in any dimension of $\Omega$ without restrictions on the form of the nonlinear function $b$, whenever (A3) is satisfied and $\bar{\varphi} \in L^{\infty}(\Gamma) . \bar{\varphi}$ is bounded and measurable if pointwise state-constraints are given only in compact subsets of $\Omega$ with the other quantities being sufficiently smooth. In twodimensional domains, pointwise state-constraints can be imposed on $\bar{\Omega}$, if $b(x, y, u)$ is linear with respect to $u$. An extension to $\bar{\varphi} \in L^{r}(\Gamma)$ requires stronger assumptions on $b$. However, we shall briefly sketch in this section that some extended form of (SSC) may partially improve the results for $n \leq 3$.

Let us assume $\bar{\varphi} \notin L^{\infty}(\Gamma)$. Then it seems to be natural to introduce in $L^{\infty}(\Gamma)$ another norm

$$
\|u\|_{\varphi}=\left(\int_{\Gamma}(1+|\bar{\varphi}(x)|) u^{2}(x) d S(x)\right)^{1 / 2} .
$$

This definition is justified, as $u \in L^{\infty}(\Gamma)$ and $y \in C(\bar{\Omega})$ holds in all parts of our paper. For $\bar{\varphi} \in L^{\infty}(\Gamma)$, the new norm is equivalent to $\|u\|_{L^{2}(\Gamma)}$. To get rid of the restrictions 
imposed on $b$ in (A4) we redefine the set of strongly active control constraints $\Gamma_{\tau}$ by

$$
\Gamma_{\tau, \varphi}=\left\{x \in \Gamma|| g_{u}(x, \bar{y}(x), \bar{u}(x))+\bar{\varphi}(x) b_{u}(x, \bar{y}(x), \bar{u}(x)) \mid \geq \tau(1+|\bar{\varphi}(x)|)\right\} .
$$

Moreover, we substitute the condition

$$
\mathcal{L}^{\prime \prime}\left(\bar{y}, \bar{u}, \bar{\varphi}, \bar{\lambda}, \bar{z}^{*}\right)\left[w_{2}, w_{2}\right] \geq \delta\left\|u_{2}\right\|_{\varphi}^{2}
$$

for (5.2). If $\bar{\varphi} \notin L^{\infty}(\Gamma)$, then (6.2) is stronger than (5.2). On the other hand, the term $\int_{\Gamma} \bar{\varphi} b_{u u} u_{2}^{2} d S$ contributes to $\mathcal{L}^{\prime \prime}$. (SSC) implies (at least) the nonnegativity of $\bar{\varphi} b_{u u}$; hence

$$
\int_{\Gamma} \bar{\varphi} b_{u u} u_{2}^{2} d S=\int_{\Gamma}|\bar{\varphi}|\left|b_{u u}\right| u_{2}^{2} d S \geq \kappa \int_{\Gamma}|\bar{\varphi}| u_{2}^{2} d S
$$

holds, provided that $\left|b_{u u}\right| \geq \kappa$. In view of this, (6.2) appears quite natural.

Now Theorem 5.2 remains true for $n \leq 3$ without assumption (A4).

This statement is easy to verify. Apart from the estimates (4.17)-(4.19), our theory is not influenced by introducing $\|u\|_{\varphi}$. The discussion of (4.17)-(4.19) is the decisive point. We are able to replace $\|\cdot\|_{L^{2}(\Gamma)}$ by $\|\cdot\|_{\varphi}$ there, as the basic inequalities (7.14)-(7.16) (Appendix 7.3) can be slightly reformulated: (7.14) is nothing more than

$$
\int_{\Gamma}|\bar{\varphi}| u^{2} d S \leq\|u\|_{\varphi}^{2}
$$

while (7.16) remains unchanged $(n=2,3)$. Only $(7.15)$ has to be substituted by

$$
\begin{aligned}
\int_{\Gamma}|\bar{\varphi}||y||u| d S & =\int_{\Gamma}|\bar{\varphi}|^{1 / 2}|y||\bar{\varphi}|^{1 / 2}|u| d S \leq\|u\|_{\varphi}\left(\int_{\Gamma}|\bar{\varphi}| y^{2} d S\right)^{1 / 2} \\
& \leq\|\bar{\varphi}\|_{L^{s /(s-2)}(\Gamma)}^{1 / 2}\|y\|_{L^{s}(\Gamma)}\|u\|_{\varphi} .
\end{aligned}
$$

Here we have invoked (7.15) for sufficiently large $s(n=2,3)$. Now a careful study of the proof of Theorem 5.2 shows that (A4) can be removed on using (6.3) and (6.4). Assuming (6.2), we arrive at the estimate (5.4) with $\|\hat{u}-\bar{u}\|_{\varphi}^{2}$ instead of $\|\hat{u}-\bar{u}\|_{L^{2}(\Gamma)}^{2}$. Then (5.4) follows from $\|u\|_{\varphi} \geq\|u\|_{L^{2}(\Gamma)}$. The same arguments apply to the first order sufficient conditions in Theorem 5.4 for $n=2$ if we redefine $L_{\beta, \tau}(\mathcal{M}, \bar{w})$ by substituting for (5.15) the inequality

$$
\left\langle\bar{z}^{*}, E^{\prime}(\bar{y}) y\right\rangle \geq-\beta \int_{\Gamma \backslash \Gamma_{\tau}}(1+|\bar{\varphi}|)|u| d S .
$$

\section{Appendix.}

7.1. On the regularity condition. Regard the state equation (4.1) linearized at $(\bar{y}, \bar{u})$. Let $\hat{Y} \subset H^{1}(\Omega)$ be the set of all solutions of this equation associated to $u \in L^{\infty}(\Gamma)$. In other words, we have $\hat{Y}=G^{\prime}(\bar{u}) L^{\infty}(\Gamma)$. (R) is satisfied in the following particular cases.

(a) $K=Z$ (no inequality constraints). Then $(\mathrm{R})$ means $F^{\prime}(\bar{y}) G^{\prime}(\bar{u}) \mathcal{C}(\bar{u})=\mathbb{R}^{m}$. This condition is satisfied if, in addition to the surjectivity property $F^{\prime}(\bar{y}) \hat{Y}=\mathbb{R}^{m}$, the following holds: There is a $\tilde{u} \in \operatorname{int}_{L^{\infty}(\Gamma)} \mathcal{U}^{\text {ad }}$ with $F^{\prime}(\bar{y}) \tilde{y}=0$. Here, $\tilde{y}$ denotes the solution of the linearized state equation (4.1) associated with $\tilde{u}-\bar{u}$, that is, 
$\tilde{y}=G^{\prime}(\bar{u})(\tilde{u}-\bar{u})$. The proof follows from [22, Lemma 1.2.2].

(b) $F=0$ (no equality constraints). In this case, $(\mathrm{R})$ is read as $E^{\prime}(\bar{y}) G^{\prime}(\bar{u}) \mathcal{C}(\bar{u})-$ $K(E(\bar{u}))=Z$. Once again, (R) is implied by two separate conditions: We assume $E^{\prime}(\bar{y}) \hat{Y}-K(E(\bar{y}))=Z$ and require the existence of an $\tilde{u} \in \operatorname{int}_{L^{\infty}(\Gamma)} \mathcal{U}^{\text {ad }}$ with the property that $E^{\prime}(\bar{y}) \tilde{y} \in K(E(\bar{y}))$ holds at $\tilde{y}=G^{\prime}(\bar{u})(\tilde{u}-\bar{u})$ [22, Lemma 1.2.2]. It should be mentioned that case (a) follows from (b).

Example. $(\mathrm{P})$ is worth discussing in this context. If the state constraint $y(0) \leq y_{0}$ is not active at $\bar{y}$, then $(\mathrm{R})$ is obviously satisfied. Therefore, we assume $\bar{y}(0)=y_{0}$ and get $K(E(\bar{y}))=\{z \in \mathbb{R} \mid z \leq 0\}=\mathbb{R}^{-}$. Then $E^{\prime}(\bar{y}) \hat{Y}-K(E(\bar{y}))=Z$ reduces to the following requirement: For every $z \in \mathbb{R}^{-}$there exists a function $u \in L^{\infty}(\Gamma)$ such that the equation $y(0)=z$ is satisfied by the corresponding solution $y$ of the linearized equation (4.4). This property is fulfilled, since we may find at least one $u \in L^{\infty}(\Gamma)$ such that $y(0) \neq 0$. Hence, $(\mathrm{R})$ is implied by the following conditions: There are $\tilde{u} \in L^{\infty}(\Gamma)$ and $\varepsilon>0$ such that $|\tilde{u}| \leq 1-\varepsilon$ holds and that the solution $\tilde{y}$ of (4.4) corresponding to $\tilde{u}-\bar{u}$ satisfies $\tilde{y}(0) \leq 0$.

(c) General case. Let us assume $\operatorname{int}_{Z} K \neq \emptyset$ and $\operatorname{int}_{L^{\infty}(\Gamma)} \mathcal{U}^{a d} \neq \emptyset$. We require the surjectivity property

$$
F^{\prime}(\bar{y}) \hat{Y}=\mathbb{R}^{m} .
$$

Moreover, assume the existence of a $\tilde{u} \in \operatorname{int}_{L^{\infty}(\Gamma)} \mathcal{U}^{\text {ad }}$ such that

$$
\begin{aligned}
E(\bar{y})+E^{\prime}(\bar{y}) \tilde{y} & \in \operatorname{int}_{Z} K, \\
F^{\prime}(\bar{y}) \tilde{y} & =0
\end{aligned}
$$

holds for $\tilde{y}=G^{\prime}(\bar{u})(\tilde{u}-\bar{u})$. Then $(\mathrm{R})$ is fulfilled. To show this, we first mention the simple fact that $\tilde{z} \in \operatorname{int}_{Z} K$ implies $\tilde{z}+z / \varrho \in K$ for arbitrary $z \in Z$ if $\varrho$ is sufficiently large. We have to verify that the system

$$
\begin{aligned}
F^{\prime}(\bar{y}) y & =z_{1}, \\
E^{\prime}(\bar{y}) y-\varrho(k-E(\bar{y})) & =z_{2}
\end{aligned}
$$

is solvable $\forall z_{1} \in \mathbb{R}^{m}, z_{2} \in Z$ by some $y \in G^{\prime}(\bar{u}) \mathcal{C}(\bar{u}), k \in K$, and $\varrho \geq 0$ : From (7.1) we find $u_{1} \in L^{\infty}(\Gamma)$ such that $y_{1}=G^{\prime}(\bar{u}) u_{1}$ solves the equation

$$
F^{\prime}(\bar{y}) y_{1}=z_{1} \text {. }
$$

Now we add to $y_{1}$ a multiple of $\tilde{y}$. Then

$$
F^{\prime}(\bar{y})\left(y_{1}+\varrho \tilde{y}\right)=F^{\prime}(\bar{y}) y_{1}=z_{1}
$$

is obtained from (7.3). Consequently, (7.4) holds for $y=y_{1}+\varrho \tilde{y}$. Moreover, we deduce from (7.2) for sufficiently large $\varrho$ that

$$
E(\bar{y})+E^{\prime}(\bar{y}) \tilde{y}-\frac{1}{\varrho}\left(z_{2}-E^{\prime}(\bar{y}) y_{1}\right)=k \in K .
$$

This relation is equivalent to

$$
E^{\prime}(\bar{y})\left(y_{1}+\varrho \tilde{y}\right)-\varrho(k-E(\bar{y}))=z_{2} .
$$

Therefore, (7.5) is satisfied by $y=y_{1}+\varrho \tilde{y}$. Furthermore, $u_{1}+\varrho(\tilde{u}-\bar{u})=\varrho\left(\tilde{u}+(1 / \varrho) u_{1}-\right.$ $\bar{u}) \in \mathcal{C}(\bar{u})$ holds for sufficiently large $\varrho$. This is true, since $\tilde{u}+(1 / \varrho) u_{1} \in \mathcal{U}^{a d}$ for $\varrho$ large enough (notice that $\tilde{u} \in \operatorname{int}_{L^{\infty}(\Gamma)} \mathcal{U}^{a d}$ ). Thus we have also shown $y \in G^{\prime}(\bar{u}) \mathcal{C}(\bar{u})$. 
7.2. Proof of the linearization theorem. To prove Theorem 4.2 we need the following auxiliary result.

Lemma 7.1. Let $\bar{u}, \hat{u} \in \mathcal{U}^{a d}$ be given with associated states $\bar{y}, \hat{y}$ defined by (2.2). Introduce $y \in Y$ as the solution of the linearized state equation

$$
\left\{\begin{aligned}
-\Delta y+y & =0 & & \text { in } \Omega \\
\partial_{\nu} y & =b_{y}(\cdot, \bar{y}, \bar{u}) y+b_{u}(\cdot, \bar{y}, \bar{u})(\hat{u}-\bar{u}) & & \text { on } \Gamma .
\end{aligned}\right.
$$

Then the estimates

$$
\begin{aligned}
\|\hat{y}-\bar{y}-y\|_{Y} & \leq C_{p}\|\hat{u}-\bar{u}\|_{L^{\infty}(\Gamma)}\|\hat{u}-\bar{u}\|_{L^{p}(\Gamma)} \quad \forall p>n-1, \\
\|\hat{y}-\bar{y}-y\|_{2} & \leq C_{2}\|\hat{u}-\bar{u}\|_{L^{\infty}(\Gamma)}\|\hat{u}-\bar{u}\|_{L^{2}(\Gamma)}
\end{aligned}
$$

are satisfied with certain constants $C_{p}, C_{2}$. If $b_{u}(x, y, u)$ does not depend on $y$ and $u$, then we have

$$
\|\hat{y}-\bar{y}-y\|_{Y} \leq C_{p}\|\hat{u}-\bar{u}\|_{L^{p}(\Gamma)}^{2} \quad \forall p>n-1 .
$$

Proof. We use the first order expansion of $b$ at $(x, \bar{y}, \bar{u})$ and obtain from (2.2), (7.6), and (4.11) the system

$$
\begin{aligned}
-\Delta(\hat{y}-\bar{y}-y)+(\hat{y}-\bar{y}-y)=0 & \text { in } \Omega, \\
\partial_{\nu}(\hat{y}-\bar{y}-y)-b_{y}(\cdot, \bar{y}, \bar{u})(\hat{y}-\bar{y}-y) & =r_{1}^{b} \quad \text { on } \Gamma,
\end{aligned}
$$

where

$$
\left|r_{1}^{b}(x)\right| \leq C_{M}\left(|\hat{y}(x)-\bar{y}(x)|^{2}+|\hat{u}(x)-\bar{u}(x)|^{2}\right)
$$

and $M$ depends on $\mathcal{U}^{\text {ad }}$ (notice that the boundedness of $\mathcal{U}^{\text {ad }}$ implies a uniform bound on all admissible states). Therefore, the discussion of (3.12) yields for $p>n-1$

$$
\begin{aligned}
\|\hat{y}-\bar{y}-y\|_{Y} & \leq c\left\|r_{1}^{b}\right\|_{L^{p}(\Gamma)} \\
& \leq c\left(\left(\int_{\Gamma}|\hat{y}-\bar{y}|^{2 p} d S\right)^{\frac{1}{p}}+\left(\int_{\Gamma}|\hat{u}-\bar{u}|^{2 p} d S\right)^{\frac{1}{p}}\right) .
\end{aligned}
$$

The mapping $u \mapsto y=G(u)$ is Lipschitz from $L^{p}(\Gamma)$ to $C(\bar{\Omega})$ for $p>n-1$. If $p=2$, then the Lipschitz property holds in the norm $\|y\|_{2}$ for $y$. For $p>n-1$, we continue by

$$
\|\hat{y}-\bar{y}-y\|_{Y} \leq c\left(\|\hat{u}-\bar{u}\|_{L^{p}(\Gamma)}^{2}+\|\hat{u}-\bar{u}\|_{L^{\infty}(\Gamma)}\|\hat{u}-\bar{u}\|_{L^{p}(\Gamma)}\right),
$$

while $p=2$ yields only

$$
\|\hat{y}-\bar{y}-y\|_{2} \leq c\|\hat{u}-\bar{u}\|_{L^{\infty}(\Gamma)}\|\hat{u}-\bar{u}\|_{L^{2}(\Gamma)} .
$$

We have shown (7.7) and (7.8). If $b_{u}$ does not depend on $(y, u)$, then $b_{u}(\cdot, \bar{y}+\vartheta(\hat{y}-$ $\bar{y}), \bar{u}+\vartheta(\hat{u}-\bar{u}))=b_{u}(\cdot, \bar{y}, \bar{u})$; hence

$$
\left|r_{1}^{b}\right|=\left|\left(b_{y}^{\vartheta}-\bar{b}_{y}\right)(\hat{y}-\bar{y})\right| \leq c|\hat{y}-\bar{y}|^{2} .
$$

This yields

$$
\left\|r_{1}^{b}\right\|_{L^{p}(\Gamma)} \leq c\left(\|\hat{y}-\bar{y}\|_{C(\Gamma)}\|\hat{y}-\bar{y}\|_{L^{p}(\Gamma)}\right) \leq c\|\hat{u}-\bar{u}\|_{L^{p}(\Gamma)}^{2},
$$


that is, (7.9).

Proof of Theorem 4.2. Define $v=\hat{u}-\bar{u}$ and let $\tilde{y}$ denote the solution of the linear system (4.1) associated to $u:=v$. We have $\tilde{y}=G^{\prime}(\bar{u}) v$, where $G: L^{\infty}(\Gamma) \rightarrow Y$ is the control-state mapping $u \mapsto y=G(u)$ for the nonlinear system (2.2). By Lemma 7.1,

$$
\|\hat{y}-\bar{y}-\tilde{y}\|_{Y} \leq e(v),
$$

where $e(v)$ denotes the right-hand side of the estimates (7.7) and (7.9), respectively, depending on the assumptions on $b$. Let us introduce the mapping $\Phi(u)=T(G(u))$. Its derivative is $\Phi^{\prime}(\bar{u}) v=T^{\prime}(\bar{y}) G^{\prime}(\bar{u}) v$, and the regularity condition (R) can be rewritten as

$$
\Phi^{\prime}(\bar{u}) \mathcal{C}(\bar{u})-\boldsymbol{K}(\Phi(\bar{u}))=\boldsymbol{Z} .
$$

We know that $\Phi(\hat{u}) \in K$, hence a Taylor expansion yields

$$
\Phi(\hat{u})=\Phi(\bar{u})+\Phi^{\prime}(\bar{u})(\hat{u}-\bar{u})+r_{1}^{\Phi},
$$

where the norm of $r_{1}^{\Phi}$ can be estimated by

$$
\left\|r_{1}^{\Phi}\right\|_{Z} \leq c e(v)
$$

Since $\Phi(\hat{u})$ and $k=\Phi(\bar{u})$ belong to $K,(7.11)$ implies $\Phi^{\prime}(\bar{u})(\hat{u}-\bar{u})+k+r_{1}^{\Phi} \in K$; thus also

$$
\Phi^{\prime}(\bar{u})(\hat{u}-\bar{u}) \in-r_{1}^{\Phi}+K(\Phi(\bar{u})) .
$$

In other words, we have $\hat{u}-\bar{u} \in \mathcal{C}(\bar{u})$ and $\Phi^{\prime}(\bar{u})(\hat{u}-\bar{u}) \leq_{K(\Phi(\bar{u}))}-r_{1}^{\Phi}$, where $z \geq_{K(\Phi(\bar{u}))}$ 0 is defined by $z \in K(\Phi(\bar{u}))$. Owing to $(\mathrm{R})$, this inequality is regular in the sense of Robinson [18]. Therefore, we are able to apply the linear version of the RobinsonUrsescu theorem (see [18]): It implies the existence of a constant $C_{R}>0$ and a $u \in \mathcal{C}(\bar{u})$ satisfying $\|u-(\hat{u}-\bar{u})\|_{L^{\infty}(\Gamma)} \leq C_{R}\left\|r_{1}^{\Phi}\right\|_{Z}$ together with

$$
\Phi^{\prime}(\bar{u}) u \in K(\Phi(\bar{u})) .
$$

Consequently, for $y=G^{\prime}(\bar{u}) u$, we have $(y, u) \in L(\mathcal{M}, \bar{w})$ and

$$
\|u-(\hat{u}-\bar{u})\|_{L^{\infty}(\Gamma)} \leq \tilde{c} e(v) .
$$

The estimates stated in (4.6) and (4.8) follow immediately.

(4.7) is proved completely analogous. Here, $e(v)$ is defined by $(7.8),\|\cdot\|_{Y}$ is to be replaced by $\|\cdot\|_{2}$, and $\|\cdot\|_{L^{2}(\Gamma)}$ is to be substituted for $\|\cdot\|_{L^{\infty}(\Gamma)}$. We rely on the continuity of $\Phi^{\prime}(\bar{y})$ in the $L^{2}$-norm.

7.3. Estimates of the Lagrange function. In this subsection we derive the estimates (4.17)-(4.19) for $r_{1}^{\mathcal{L}}, r_{2}^{\mathcal{L}}$, and $\mathcal{L}^{\prime \prime}$. They depend mainly on the estimation of $I$ defined in (4.10), which is performed by the discussion of the following integrals:

$$
\int_{\Gamma}|\bar{\varphi}| u^{2} d S \leq c\|u\|_{L^{2}(\Gamma)}^{2}
$$

provided that assumption (A4 (i)) is fulfilled, and 


$$
\begin{aligned}
\int_{\Gamma}|\bar{\varphi}||y||u| d S & \leq c\|\bar{\varphi} y\|_{L^{2}(\Gamma)}\|u\|_{L^{2}(\Gamma)} \leq c\left\|\bar{\varphi}^{2}\right\|_{L^{(s / 2)^{\prime}(\Gamma)}}^{1 / 2}\left\|y^{2}\right\|_{L^{s / 2}(\Gamma)}^{1 / 2}\|u\|_{L^{2}(\Gamma)} \\
& \leq c\|\bar{\varphi}\|_{L^{2 s /(s-2)}(\Gamma)}\|y\|_{L^{s}(\Gamma)}\|u\|_{L^{2}(\Gamma)} .
\end{aligned}
$$

These estimates are justified by (A4 (ii)): For $n=2$ we know $y \in C(\Gamma)$ and $\varphi \in L^{r}(\Gamma)$ $\forall r<\infty$. If $n \geq 3$, then $y \in L^{s}(\Gamma)$ holds $\forall s<2(n-1) /(n-3)$ (including $s<\infty$ for $n=3)$. The function $2 s /(s-2)=2 /(1-1 / s)$ is monotone decreasing. Therefore, $s \uparrow 2(n-1) /(n-3)$ implies $2 s /(s-2) \downarrow n-1$, so that $\bar{\varphi} \in L^{r}(\Gamma)$ for some $r>n-1$ justifies (7.15) with a sufficiently large $s$. Finally,

$$
\int_{\Gamma}|\bar{\varphi}| y^{2} d S \leq\|\bar{\varphi}\|_{L^{(s / 2)^{\prime}(\Gamma)}}\left\|y^{2}\right\|_{L^{s / 2}(\Gamma)}=\|\bar{\varphi}\|_{L^{s /(s-2)}(\Gamma)}\|y\|_{L^{s}(\Gamma)}^{2}
$$

is estimated by (A4 (iii)): In the case $n=2$ we can take $s=\infty$, as $y \in C(\Gamma)$ and $\varphi \in L^{1}(\Gamma)$ is true without any additional assumption. For $n=3$ we know $y \in L^{s}(\Gamma) \forall s<\infty$. If $s \uparrow \infty$, then $s /(s-2) \downarrow 1<n /(n-1)$. Since $\varphi \in L^{r}(\Gamma)$ holds $\forall r<n /(n-1),(7.16)$ is true for sufficiently large $s$. In the case $n \geq 4$ we repeat the analysis of the case $n \geq 3$. This leads to the additional assumption $\bar{\varphi} \in L^{r}(\Gamma)$ for some $r>\frac{n-1}{2}$. Now it is easy to derive the estimates (4.17)-(4.19) for $\mathcal{L}^{\prime \prime}, r_{1}^{\mathcal{L}}$, and $r_{2}^{\mathcal{L}}$ : For instance, $I$ in $(4.10)$ is handled by (7.14)-(7.16), and

$$
\begin{aligned}
|I| & \leq \int_{\Gamma}|\bar{\varphi}|\left(\left|\bar{b}_{y y}\right|\left|y_{1} y_{2}\right|+\left|\bar{b}_{y u}\right|\left(\left|y_{1} u_{2}\right|+\left|y_{2} u_{1}\right|\right)+\left|\bar{b}_{u u}\right|\left|u_{1} u_{2}\right| d S\right. \\
& \leq c\left(\left\|y_{1}\right\|_{2}+\left\|u_{1}\right\|_{L^{2}(\Gamma)}\right)\left(\left\|y_{2}\right\|_{2}+\left\|u_{2}\right\|_{L^{2}(\Gamma)}\right),
\end{aligned}
$$

as $\bar{b}_{y y}, \bar{b}_{y u}$, and $\bar{b}_{u u}$ belong to $L^{\infty}(\Gamma)$. The other parts of $\mathcal{L}^{\prime \prime}$ are discussed by means of (A1)-(A3). This yields (4.19) after easy evaluations. In the same way, the remainder terms are investigated. Here, the quantities in $I$ are the most difficult ones again. For instance, (7.14)-(7.16) applies to discussing

$$
\begin{aligned}
\left|r_{2}^{I}\right|= & \int_{\Gamma}|\bar{\varphi}|\left\{\left|b_{y y}^{\vartheta}-\bar{b}_{y y}\right||y-\bar{y}|^{2}+2\left|b_{y u}^{\vartheta}-\bar{b}_{y u}\right||y-\bar{y}||u-\bar{u}|\right. \\
& \left.\quad+\left|b_{u u}^{\vartheta}-\bar{b}_{u u}\right||u-\bar{u}|^{2}\right\} d S \\
\leq & c \eta\left(\|y-\bar{y}\|_{C(\Gamma)}+\|u-\bar{u}\|_{L^{\infty}(\Gamma)}\right)\left(\|y-\bar{y}\|_{2}^{2}+\|u-\bar{u}\|_{L^{2}(\Gamma)}^{2}\right)
\end{aligned}
$$

which contributes to $r_{2}^{\mathcal{L}}$. The other terms of $r_{2}^{\mathcal{L}}$ are handled by the estimates for second order derivatives in (A1)-(A3) in a direct way. Simple evaluations of this type verify (4.17)-(4.18). We leave the details to the reader.

\section{REFERENCES}

[1] R. A. Adams, Sobolev Spaces, Academic Press, New York, NY, 1978.

[2] W. Alt, On the approximation of infinite optimization problems with an application to optimal control problems, Appl. Math. Optim., 12 (1984), pp. 15-27.

[3] W. Alt, The Lagrange-Newton method for infinite-dimensional optimization problems, Numer. Funct. Anal. Optim., 11 (1990), pp. 201-224.

[4] W. Alt, Discretization and mesh-independence of Newton's method for generalized equations, in Mathematical Programming with Data Perturbations, Lecture Notes in Pure Appl. Math. 195, A. K. Fiacco, ed., Dekker, New York, 1998, pp. 1-30.

[5] F. Bonnans Second order analysis for control constrained optimal control problems of semilinear elliptic systems, Appl. Math. Optim., 38 (1998), pp. 303-325. 
[6] F. Bonnans, and F. CASas, Contrôle de systémes elliptiques semilinéaires comportant des contraintes sur l'état, Nonlinear Partial Differential Equations and Their Applications, H. Brezis and J. L. Lions, eds., College de France Seminar Vol. VIII; Pitman Res. Notes Math. Ser. 166, Longman, Harlow, UK, 1988, pp. 69-86.

[7] E. CASAS, Boundary control of semilinear elliptic equations with pointwise state constraints, SIAM J. Control Optim., 31 (1993), pp. 993-1006.

[8] E. Casas, And F. Tröltzsch, Second order necessary optimality conditions for some stateconstrained control problems of semilinear elliptic equations, Appl. Math. Optim., 39 (1999), pp. 211-228.

[9] E. Casas, F. Tröltzsch, and A. Unger, Second order sufficient optimality conditions for a nonlinear elliptic control problem, Z. Anal. Anwendungen, 15 (1996), pp. 687-707.

[10] A. L. Dontchev, W. W. Hager, A. B. Poore, and B. Yang, Optimality, stability, and convergence in nonlinear control, Appl. Math. Optim., 31 (1995), pp. 297-326.

[11] H. Goldberg And F. Tröltzsch, Second order optimality conditions for a class of control problems governed by non-linear integral equations with applications to parabolic boundary control, Optim., 20 (1989), pp. 687-698.

[12] H. Goldberg And F. Tröltzsch, Second-order sufficient optimality conditions for a class of nonlinear parabolic boundary control problems, SIAM J. Control Optim., 31 (1993), pp. 1007-1025.

[13] A. D. Ioffe, Necessary and sufficient conditions for a local minimum. III. Second order conditions and augmented duality, SIAM J. Control Optim., 17 (1979), pp. 266-288.

[14] H. MAURER, First and second order sufficient optimality conditions in mathematical programming and optimal control, Math. Programming Study, 14 (1981), pp. 163-177.

[15] H. Maurer AND J. Zowe, First- and second-order conditions in infinite-dimensional programming problems, Math. Programming, 16 (1979), pp. 98-110.

[16] M. K. V. Murthy and G. Stampacchia, A variational inequality with mixed boundary conditions, Israel J. Math., 13 (1972), pp. 188-224.

[17] J. NeČAs, Les Méthodes Directes en Théorie des Equations Elliptiques, Editeurs Academia, Prague, 1967.

[18] S. M. Robinson, Stability theory for systems of inequalities. I. Linear systems, SIAM J. Numer. Anal., 12 (1975), pp. 754-769.

[19] G. Stampacchia, Problemi al contorno ellittici con dati discontinui dotati di soluzioni Hölderiane, Ann. Math. Pura Appl., 51 (1960), pp. 1-38.

[20] G. Stampacchia, Equations Elliptiques du Second Ordre à Coefficients Discontinus, Les Presses de l'Université de Montreal, Montreal, 1966.

[21] H. Triebel, Interpolation Theory, Function Spaces, Differential Operators, 2nd ed., J. A. Barth Verlag, Heidelberg, 1995.

[22] F. Tröltzsch, Optimality Conditions for Parabolic Control Problems and Applications, Teubner-Texte Math. 62, B. G. Teubner Verlagsgesellschaft, Leipzig, 1984.

[23] J. Zowe And S. Kurcyusz, Regularity and stability for the mathematical programming problem in Banach spaces, Appl. Math. Optim., 5 (1979), pp. 49-62. 